The online version of this article is published within an Open Access environment subject to the conditions of the Creative Commons Attribution-NonCommercial-ShareAlike licence <http:// creativecommons.org/licenses/by-nc-sa/3.0/>. The written permission of Cambridge University Press must be obtained for commercial re-use.

doi: $10.1017 / \mathrm{jfm} .2013 .192$

\title{
Spectral non-locality, absolute equilibria and Kraichnan-Leith-Batchelor phenomenology in two-dimensional turbulent energy cascades
}

\author{
B. H. Burgess ${ }^{1, \dagger}$ and T. G. Shepherd ${ }^{1,2}$ \\ ${ }^{1}$ Department of Physics, University of Toronto, Toronto, ON, Canada M5S 1A7 \\ ${ }^{2}$ Department of Meteorology, University of Reading, Reading, Berkshire RG6 6BB, UK
}

(Received 3 October 2012; revised 9 April 2013; accepted 10 April 2013; first published online 14 May 2013)

\begin{abstract}
We study the degree to which Kraichnan-Leith-Batchelor (KLB) phenomenology describes two-dimensional energy cascades in $\alpha$ turbulence, governed by $\partial \theta / \partial t+$ $J(\psi, \theta)=v \nabla^{2} \theta+f$, where $\theta=(-\Delta)^{\alpha / 2} \psi$ is generalized vorticity, and $\hat{\psi}(\boldsymbol{k})=k^{-\alpha} \hat{\theta}(\boldsymbol{k})$ in Fourier space. These models differ in spectral non-locality, and include surface quasigeostrophic flow $(\alpha=1)$, regular two-dimensional flow $(\alpha=2)$ and rotating shallow flow $(\alpha=3)$, which is the isotropic limit of a mantle convection model. We re-examine arguments for dual inverse energy and direct enstrophy cascades, including Fjørtoft analysis, which we extend to general $\alpha$, and point out their limitations. Using an $\alpha$-dependent eddy-damped quasinormal Markovian (EDQNM) closure, we seek self-similar inertial range solutions and study their characteristics. Our present focus is not on coherent structures, which the EDQNM filters out, but on any self-similar and approximately Gaussian turbulent component that may exist in the flow and be described by KLB phenomenology. For this, the EDQNM is an appropriate tool. Nonlocal triads contribute increasingly to the energy flux as $\alpha$ increases. More importantly, the energy cascade is downscale in the self-similar inertial range for $2.5<\alpha<10$. At $\alpha=2.5$ and $\alpha=10$, the KLB spectra correspond, respectively, to enstrophy and energy equipartition, and the triad energy transfers and flux vanish identically. Eddy turnover time and strain rate arguments suggest the inverse energy cascade should obey KLB phenomenology and be self-similar for $\alpha<4$. However, downscale energy flux in the EDQNM self-similar inertial range for $\alpha>2.5$ leads us to predict that any inverse cascade for $\alpha \geqslant 2.5$ will not exhibit KLB phenomenology, and specifically the KLB energy spectrum. Numerical simulations confirm this: the inverse cascade energy spectrum for $\alpha \geqslant 2.5$ is significantly steeper than the KLB prediction, while for $\alpha<2.5$ we obtain the KLB spectrum.
\end{abstract}

Key words: isotropic turbulence, turbulent flows, turbulence theory 


\section{Introduction}

Generalized two-dimensional (2D) fluid dynamics, also known as $\alpha$ turbulence, was introduced by Pierrehumbert, Held \& Swanson (1994) as a tool for studying the effects of spectral non-locality on turbulence. With forcing and dissipation, the dynamics are governed by

$$
\frac{\partial \theta}{\partial t}+J(\psi, \theta)=v \nabla^{2} \theta+f,
$$

where $J(\psi, \theta)$ is the 2D Jacobian. The streamfunction $\psi$ and generalized vorticity $\theta$ are related in real space by $\theta=(-\Delta)^{\alpha / 2} \psi$, where $(-\Delta)^{\alpha / 2}$ is the fractional Laplacian, and in Fourier space by

$$
\hat{\psi}(\boldsymbol{k})=|\boldsymbol{k}|^{-\alpha} \hat{\theta}(\boldsymbol{k}) \equiv k^{-\alpha} \hat{\theta}(\boldsymbol{k}),
$$

where $k$ is the 2D wavenumber, and $\hat{\psi}(\boldsymbol{k})$ and $\hat{\theta}(\boldsymbol{k})$ are Fourier coefficients of the streamfunction and generalized vorticity, respectively. For $\alpha=2$, the generalized vorticity is the familiar $\omega=-\nabla^{2} \psi$, where we use Batchelor's sign convention (Batchelor 1969), and the system reduces to 2D Navier-Stokes flow as a special case. For larger positive $\alpha$, the contribution of the large- $k$ generalized vorticity modes to the streamfunction is preferentially suppressed. As a result, $\psi$ has a higher degree of spatial smoothing relative to $\theta$, and we may expect the dynamics to be more spectrally non-local, all else being equal. The degree of spectral non-locality has an impact on the validity of Kraichnan-Leith-Batchelor (KLB) self-similar inertial range phenomenology (Kraichnan 1967; Leith 1968; Batchelor 1969), error growth and predictability times, and the possibility of dynamical subgrid-scale parameterizations, so it is of practical as well as theoretical interest.

Several of these $\alpha$ turbulence models are geophysically relevant. Shallow-water quasigeostrophic dynamics in the limit of strong rotation, i.e. the asymptotic model limit of the Charney-Hasegawa-Mima equation (Larichev \& McWilliams 1991; Iwayama, Shepherd \& Watanabe 2002) corresponds to $\alpha=-2$ (Smith et al. 2002). Surface quasigeostrophic (SQG) dynamics, a simplified model for edge waves on the tropopause or temperature advection near the Earth's surface (Blumen 1978; Held et al. 1995), is given by $\alpha=1$, and 2D Navier-Stokes dynamics corresponds to $\alpha=2$, as noted above. The 'rotating shallow flow' (RSF) equation (Tran 2004) is given by $\alpha=3$, and is the isotropic limit of a mantle convection model, wherein a thin fluid on a rotating domain is driven by uniform internal heating and heated from below (Weinstein, Olson \& Yuen 1989). For $\alpha>3$, the azimuthal velocity induced by a point vortex increases with distance (Iwayama \& Watanabe 2010), which calls into question the physical relevance of these systems. However, all values of $\alpha$, including non-integer values, give, at least in principle, well-defined systems, and the entire family is of interest when studying spectral non-locality, since this varies continuously with $\alpha$.

For $\alpha \geqslant 2$, the downscale cascade of generalized enstrophy, $Z=\overline{\theta^{2}} / 2$, where the overline represents a domain average, is known to be dominated by spectrally nonlocal interactions, with ultralocal transfers mediated by a distant infrared wavenumber (Watanabe \& Iwayama 2007; Tran, Dritschel \& Scott 2010). For $\alpha=2$, logarithmic corrections to the Kolmogorov spectrum in the enstrophy cascade were suggested early on (Kraichnan 1967, 1971b), and for $\alpha>2$ the enstrophy spectrum shallows to $k^{-1}$ (Pierrehumbert et al. 1994; Schorghofer 2000; Watanabe \& Iwayama 2004), 
characteristic of a passive tracer in the non-local, infinite Prandtl number regime (Batchelor 1959).

In the generalized energy cascading subrange, for $\alpha<4$, the strain rate at a given $k$ associated with the KLB spectrum is dominated by local contributions, which led Pierrehumbert et al. (1994) to conclude that this cascade is spectrally local for $\alpha<4$. For $\alpha=2$, the energy cascade proceeds toward larger scales. In this inverse-cascading case, strain rate arguments for spectral locality are based on 'negative eddy viscosity', i.e. the notion that strain thins smaller-scale vortices, thereby transferring energy upscale (Kraichnan 1976).

However, the mechanism of the Navier-Stokes inverse cascade is much debated. Depending on the dynamics generating upscale energy transfer, spectral locality of the strain rate may not indicate local transfers. It has been suggested that the Navier-Stokes energy cascade may proceed via aggregation of like-sign vortices (Paret \& Tabeling 1997, 1998), in which case it might have little to do with shear or strain. Other results (Chen et al. 2006; Xiao et al. 2009) support the notion that the inverse cascade in forced Navier-Stokes turbulence is due to vortex thinning in eddy-eddy interactions involving comparable scales, but that thinning is due to turbulent stress from small-scale strain rotated by $45^{\circ}$. A consequence is that the cascade is only weakly local, because strain and skew strain are orthogonal at the same scale.

Recent numerical results (Scott 2007; Vallgren 2011) demonstrate that the energy spectrum in the Navier-Stokes $(\alpha=2)$ inverse energy cascade steepens from $k^{-5 / 3}$ to $k^{-2}$ when the forcing scale is adequately resolved. The spectral steepening was attributed to coherent vortex formation. Vallgren (2011) also found numerically that upscale energy flux was entirely due to non-local interactions, with a small downscale flux due to local triads, and weak but persistent coupling between inverse and direct cascade scales. These results are consistent with Kraichnan's early observation that for 2D Navier-Stokes turbulence energy transfers are much less local than in threedimensional turbulence (Kraichnan 1971b). It is important to emphasize that for forcing sufficiently close to the dissipation range, inverse cascades with the KLB spectrum $k^{-5 / 3}$ are realized. Moreover, when coherent vortices are filtered out of the vorticity field, the turbulent background is found to retain the KLB spectrum (Scott 2007; Vallgren 2011), indicating that the steeper spectrum is due to the presence of vortices, and that the KLB theory does describe the background.

Herein we study the applicability of KLB inertial range phenomenology to 2D fluids using several analytical approaches, as well as numerical simulations. We focus on $\alpha>0$ and the generalized energy cascade, which has not been as thoroughly studied as the generalized enstrophy cascade. We examine the validity of arguments used to motivate expectations of dual energy-enstrophy cascades, and to predict when KLB inertial range phenomenology should be valid. Our goal is to explore parameter space by varying $\alpha$ to see whether, where and why standard phenomenology, which otherwise might be expected to apply, breaks down. We do not address coherent vortices; our focus, rather, is on the turbulent background, which is known to exhibit the KLB energy spectrum at least for $\alpha=2$.

In $\S 2$, we review the basics of $\alpha$ turbulence. We then re-examine in $\S 3$ arguments for inverse energy transfer and direct enstrophy transfer, pointing out their limitations. In $\S 4$, we focus in particular on Fjørtoft arguments (Fjørtoft 1953), which we extend to $\alpha$ turbulence. Following Merilees \& Warn (1975), we study how the percentages of energy and enstrophy exchanged with smaller and larger scales depend on triad geometry, and how this varies with $\alpha$. 
We then generalize the eddy-damped quasinormal Markovian (EDQNM) closure (Orszag 1970) to $\alpha$ turbulence in $\S 5$, seek self-similar inertial range solutions and study their properties, such as transfer non-locality and cascade directions. The EDQNM is a Markovianized closure related to the direct interaction approximation (DIA) (Kraichnan 1959), a statistical closure that corresponds to the lowest order of formally exact classical renormalized perturbation theory (Martin, Siggia \& Rose 1973). The EDQNM involves closing the moment hierarchy at second order, and is an approximate model for the average behaviour of an ensemble of turbulent flows. It is in a sense the simplest model of turbulence, preserving enough non-Gaussianity to represent turbulent transfers, but not so much as to be analytically intractable. The EDQNM is systematically derived, conserves quadratic invariants, is realizable and self-consistent (Bowman, Krommes \& Ottaviani 1993).

Since it closes the moment hierarchy in terms of the second-order correlation function, the EDQNM does not capture coherent structures, which are associated with higher-order statistics. Nor does it capture any effects these structures have on inertial range phenomenology, such as steepening of the energy spectrum past the KLB prediction in the presence of coherent vortices. Herring \& McWilliams (1985) found that a related closure, the test-field model (TFM), was only quantitatively accurate if the random forcing was strong enough to disrupt coherent vortex formation. The TFM was most accurate for strong forcing at low wavenumbers, producing an enstrophy inertial range. Forcing at higher wavenumbers produced an inverse energy cascade in which coherent vortices formed. These vortices did not appreciably cascade energy toward large scales, and as a result, the TFM overestimated the flux in the inverse cascade.

As the simplest models of turbulence, closures can be used to test heuristic phenomenological arguments, which generally make no assumptions about coherent vortices, regarding spectral non-locality and the validity of KLB theory. Furthermore, as stated above, our interest here is not in coherent vortices, but rather the various characteristics of self-similar inertial range solutions for the energy cascade, such as the degree to which transfers are spectrally non-local and the directions of the fluxes. We compare our results with classical predictions for cascade directions and the applicability of KLB theory, with a view to gaining deeper insight into turbulent energy transfers, flux directions and the conditions under which KLB theory can be expected to apply.

Finally, we explore the implications of our findings for the applicability of KLB phenomenology to inverse energy cascades in generalized 2D fluids. Numerical simulations, presented in $\S 8$, support our predictions based on the EDQNM closure.

\section{Quadratic invariants and inertial ranges}

In the absence of forcing and dissipation, equation (1.1) has two quadratic invariants, the generalized energy $E$ and the generalized enstrophy $Z$,

$$
E \equiv \frac{1}{2} \overline{\psi \theta}=\int_{0}^{\infty} \mathscr{E}(k) \mathrm{d} k, \quad Z \equiv \frac{1}{2} \overline{\theta^{2}}=\int_{0}^{\infty} \mathscr{Z}(k) \mathrm{d} k,
$$

where we have assumed isotropy. Note that $E \geqslant 0$ for all $\alpha$ by virtue of (1.2). We will henceforth refer to these invariants as energy and enstrophy for brevity. These quantities are intensive, i.e. spatial averages, as denoted by the overbar. By virtue of 
(1.2), the spectra are related by

$$
\mathscr{Z}(k)=k^{\alpha} \mathscr{E}(k) .
$$

For $\alpha>0$, the dimensions of $\psi$ and $\theta$ are $\left[L^{2} T^{-1}\right]$ and $\left[L^{2-\alpha} T^{-1}\right]$, respectively. The velocity field remains related to $\psi$ in the usual way, $\boldsymbol{v}=-\hat{\boldsymbol{z}} \times \nabla \psi$. Assuming $k$-independent fluxes, and that the spectra depend only on the local wavenumber and flux (i.e. making Kolmogorov's locality hypothesis), dimensional analysis yields

$$
\begin{aligned}
& \mathscr{E}(k)=C \varepsilon^{2 / 3} k^{-(7-\alpha) / 3}, \\
& \mathscr{E}(k)=C^{\prime} \eta^{2 / 3} k^{-(7+\alpha) / 3},
\end{aligned}
$$

for the energy spectrum in the energy and enstrophy cascades, respectively. Here $C$ and $C^{\prime}$ are dimensionless constants, $\varepsilon$ and $\eta$ are fluxes of energy and enstrophy, and we have used the fact that $\mathscr{E}(k)$ has dimension $\left[L^{5-\alpha} T^{-2}\right]$. The KLB spectra $(2.3 a)$ and (2.3b) can alternatively be derived using scaling symmetry, which immediately applies to $\alpha<0$ as well. (See appendix A.)

Dimensional analysis yields a local eddy turnover time

$$
\tau(k)=\left[k^{5-\alpha} \mathscr{E}(k)\right]^{-1 / 2} .
$$

In the enstrophy range $\tau(k) \propto k^{-(4-2 \alpha) / 3}$, so that $\tau$ is $k$-independent for $\alpha=2$ and grows with $k$ for $\alpha>2$. An eddy turnover time increasing with $k$ means smallerscale eddies take longer to evolve than larger eddies, which seems unphysical, and suggests the need for a non-locally corrected spectrum. In the energy inertial range, $\tau(k) \propto k^{-(4-\alpha) / 3}$, so $\tau$ is $k$-independent for $\alpha=4$ and a decreasing function of $k$ for $\alpha<4$.

The strain rate at wavenumber $k$ is

$$
\begin{aligned}
S(k) & =\left[\int_{k_{0}}^{k} \mathscr{E}\left(k^{\prime}\right)\left(k^{\prime}\right)^{4-\alpha} \mathrm{d} k^{\prime}\right]^{1 / 2}=\left[\int_{k_{0}}^{k} \mathscr{E}\left(k^{\prime}\right)\left(k^{\prime}\right)^{5-\alpha} d \log k^{\prime}\right]^{1 / 2} \\
& \sim\left\{\int_{k_{0}}^{k}\left[\tau\left(k^{\prime}\right)\right]^{-2} \mathrm{~d} \log k^{\prime}\right\}^{1 / 2},
\end{aligned}
$$

where $k_{0}^{-1}$ is the energy-containing scale and the primes denote the dummy integration variable. When $\tau(k)$ becomes $k$-independent, each wavenumber octave makes an equal contribution, and the strain rate becomes non-locally dominated. For $\alpha<2$ and $\alpha<4$ the strain rate is locally dominated in the enstrophy and energy cascades, respectively. The behaviour of $\tau(k)$ and $S(k)$ seems to suggest that the energy-cascading inertial range does not become spectrally non-local until $\alpha=4$ (Pierrehumbert et al. 1994), and that one should observe the spectrum (2.3a) in this inertial range for $\alpha<4$.

Assuming that any initial error is transferred from scale $k$ to scale $k / 2$ in a time proportional to $\tau(k)$ (i.e. that the error cascade is logarithmic), one obtains a predictability time (Vallis 1985)

$$
T(k)=\int_{k}^{\infty} \tau\left(k^{\prime}\right) \mathrm{d} \ln k^{\prime} \sim \int_{k}^{\infty} k^{\prime-(7-\alpha-n) / 2} \mathrm{~d} k^{\prime},
$$

where (2.4) has been used and we have set $\mathscr{E}(k) \propto k^{-n}$. The integral converges, yielding a finite predictability time, if $(7-\alpha-n) / 2>1$. Substituting $n=(7-\alpha) / 3$, one obtains finite predictability for $\alpha<4$ in the KLB energy cascade. In the KLB enstrophy cascade, $n=(7+\alpha) / 3$ yields finite predictability for $\alpha<2$. The 
mathematical reason for this is clear: when the eddy turnover time becomes $k$ independent, the integrand of (2.6) is proportional to $k^{-1}$, and the predictability time becomes infinite. This makes intuitive sense: the error cascade proceeds at the same rate at high and low wavenumbers for a scale-independent eddy turnover time. For an eddy turnover time decreasing like some inverse power of $k$, the cascade proceeds much faster at higher wavenumbers, transferring error to large scales more quickly and translating into finite predictability.

We note that the spectra (2.3a) and (2.3b) can also be derived using a variational principle that involves minimizing the predictability time subject to energy and enstrophy conservation (Benzi, Vitaletti \& Vulpiani 1978). This corresponds to maximally chaotic dynamics, in the sense that interactions between different scales are maximized.

\section{Arguments for cascade directions}

A number of arguments, which can be generalized to $\alpha$ turbulence, imply net transfer of energy toward large scales and of enstrophy toward small scales in 2D fluid flows. As a result, turbulent dual cascades, with coexisting inverse energy and direct enstrophy inertial ranges, are possible. These arguments include a Batchelor-style similarity argument, arguments relying on global conservation of energy and enstrophy, and Fjørtoft arguments, which rely on dual conservation of energy and enstrophy in wavevector triad interactions. Fjørtoft arguments will be examined in greater detail in $\S 4$.

The first two arguments examined in this section are for spectrally localized disturbances spreading in wavenumber space. The first, a Batchelor-style similarity argument (Batchelor 1969; Vallis 2006), is for a freely decaying fluid with some initial spectrum. It assumes that energy is conserved and that enstrophy decreases to zero as $t \rightarrow \infty$, so that the energy is the only relevant parameter. One posits a similarity form for the energy spectrum, and then energy conservation implies that the energy centroid wavenumber moves to larger scales as time increases (Batchelor 1969). This argument relies on the similarity assumption, an initially spectrally localized distribution, assumed energy conservation and enstrophy dissipation.

A second argument relies on dual global conservation of energy and enstrophy, and on the assumption that a spectrally localized initial energy and enstrophy distribution spreads out in spectral space (Rhines 1975, 1979; Salmon 1988; Vallis 2006). This argument is for inviscid unforced decaying turbulence, and it was extended to general $\alpha$ by Smith et al. (2002). Global conservation together with the assumption that the energy and enstrophy distributions broaden around the initial wavenumber allow one to prove that the energy and enstrophy centroid wavenumbers move to larger and smaller scales, respectively, for $\alpha>0$.

It is important to note that both the Batchelor similarity argument and the energy-enstrophy conservation argument assume an initially localized and spreading spectral distribution of energy, which is not statistically steady. As such, even though these arguments have been used to motivate dual cascade predictions in 2D turbulence, they do not strictly apply to statistically steady inertial ranges, in particular the infiniteReynolds-number ideal inertial ranges for which classical KLB phenomenology was developed.

Gkioulekas \& Tung (2007) proved that in statistically stationary 2D turbulence $(\alpha=2)$ with narrow-band forcing energy is transferred to larger scales and enstrophy to smaller scales. Their proof relies on the dissipation terms, which include both 
hyperviscosity and hypoviscosity (i.e. dissipation at both small and large scales), and they derive integral constraints that imply energy fluxes upscale and enstrophy downscale in the net. They extend the proof to $\alpha=1$ (SQG) and to time-dependent unforced turbulence with a compactly supported initial distribution spreading in spectral space. (Note that what they refer to as 'energy' in SQG dynamics we here refer to as generalized enstrophy, which for the SQG model coincides with kinetic energy.)

For Navier-Stokes turbulence $(\alpha=2)$, there is ample numerical evidence that $E$ and $Z$ do indeed cascade toward large and small scales, respectively, in accordance with the above arguments. However, simultaneous dual cascades with KLB energy spectral slopes $-5 / 3$ and -3 have proved elusive, suggesting that such a dual cascade is not generic. At the least, the statistics of both the energy and enstrophy cascades are sensitive to the forcing and dissipation details. In simulations forced at intermediate scales, and where an inverse energy cascade is present, the spectral slope in the enstrophy cascade is generally steeper than the KLB prediction, although evidence suggests it tends to -3 in the limit of infinite Reynolds number (Boffetta \& Musacchio 2010). Farazmand et al. (2011) did obtain dual cascades with the KLB spectra, but they used an optimal forcing unlikely to be spontaneously realized.

The energy cascade is also sensitive to the forcing details, and possibly to the presence of an enstrophy cascade: when the forcing scales are well-resolved and in the absence of large-scale drag, coherent structures form in the inverse energy cascade, resulting in steepening of the total energy spectrum (Scott 2007; Vallgren 2011). However, in numerical simulations with small-scale forcing sufficiently near the dissipation range, an inverse energy cascade with the KLB spectrum $\mathscr{E} \propto k^{-5 / 3}$ develops. Even in the presence of coherent vortices the turbulent background flow obtained by filtering out coherent vortices retains the KLB spectrum $k^{-5 / 3}$ (Borue 1994; Scott 2007; Vallgren 2011). Whether there is a turbulent background with the KLB spectrum for values of $\alpha$ other than $\alpha=2$ is not known. In $\S 5$ we investigate this issue analytically and in $\S 8$ we present preliminary numerical results.

\section{Fjørtoft arguments}

Fjørtoft arguments (Fjørtoft 1953) use dual conservation of energy and enstrophy in wavevector triad interactions to study what percentages of energy and enstrophy are exchanged with smaller and larger scales by triads of various geometries. Based on such arguments, Fjørtoft (1953) made his famous statement that any transfer of energy toward small scales in 2D Navier-Stokes flow must be accompanied by transfer of still more energy toward large scales.

Merilees \& Warn (1975) pointed out that this statement was in error: the percentages of energy exchanged with large and small scales depend on triad geometry, so one cannot draw conclusions about net energy flow based on examining one triad. In a more detailed analysis, they found that $70 \%$ of triads in 2D Navier-Stokes flow $(\alpha=2)$ exchange more energy with large scales, while 30\% exchange more energy with small scales. Here, we extend their analysis to $\alpha$ turbulence models to see how the fraction of triads exchanging most of their energy with large scales varies with $\alpha$.

Fjørtoft arguments have been used to motivate expectations that energy should cascade toward larger scales and enstrophy toward smaller scales in the net in 2D turbulence. However, as has been pointed out before (Kraichnan 1967; Merilees \& Warn 1975; Tung \& Welch 2001; Gkioulekas \& Tung 2007), the triad conservation laws alone are insufficient to determine the directions of energy and enstrophy flow. 
One needs dissipation or an additional assumption to set the direction of time. For instance, Kraichnan (1967) defines the time arrow by assuming the system tries to go to absolute thermodynamic equilibrium. The triad conservation laws also yield no information on how much various triad shapes contribute to the fluxes, i.e. how dynamically active various triads are, which is important because, as noted in the previous paragraph, some triads transfer more energy upscale and others more energy downscale, and likewise with enstrophy.

We work in a domain of side length $L$ with periodic boundary conditions, and expand the streamfunction and (generalized) vorticity in Fourier series,

$$
\begin{gathered}
\psi(\boldsymbol{x}, t)=\sum_{\boldsymbol{k}} \hat{\psi}(\boldsymbol{k}, t) \mathrm{e}^{\mathrm{i} \boldsymbol{k} \cdot \boldsymbol{x}}, \\
\theta(\boldsymbol{x}, t)=\sum_{\boldsymbol{k}} k^{\alpha} \hat{\psi}(\boldsymbol{k}, t) \mathrm{e}^{\mathrm{i} \boldsymbol{k} \cdot \boldsymbol{x}}=\sum_{k} \hat{\theta}(\boldsymbol{k}, t) \mathrm{e}^{\mathrm{i} \boldsymbol{k} \cdot \boldsymbol{x}},
\end{gathered}
$$

where $\boldsymbol{k}=2 \pi\left(k_{x}, k_{y}\right) / L$. Substituting (4.1) and (4.2) into (1.1) and neglecting forcing and dissipation yields the Fourier space version of the inviscid governing equation,

$$
\frac{\partial \hat{\theta}(\boldsymbol{k}, t)}{\partial t}=\frac{1}{2} \sum_{\boldsymbol{p}} \sum_{\boldsymbol{q}} \frac{\left(q^{\alpha}-p^{\alpha}\right)(\boldsymbol{p} \times \boldsymbol{q})_{z}}{p^{\alpha} q^{\alpha}} \delta_{\boldsymbol{p}+\boldsymbol{q}-\boldsymbol{k}} \hat{\theta}(\boldsymbol{p}, t) \hat{\theta}(\boldsymbol{q}, t),
$$

where the factor of $1 / 2$ on the right-hand side comes from symmetrization of the sum in $\boldsymbol{p}$ and $\boldsymbol{q}$, and $\delta_{\boldsymbol{p}+\boldsymbol{q}-\boldsymbol{k}}$ is the Kronecker delta, since we are in a finite domain.

Multiplying (4.3) through by $\hat{\theta}^{*}(\boldsymbol{k}, t)=\hat{\theta}(-\boldsymbol{k}, t)$, which holds for real fields, multiplying the complex conjugate of $(4.3)$ by $\hat{\theta}(\boldsymbol{k}, t)$, and adding, we obtain the governing equation for the enstrophy in wavenumber $k$,

$$
\begin{aligned}
\frac{\partial}{\partial t}|\hat{\theta}(\boldsymbol{k}, t)|^{2}= & \frac{1}{2} \sum_{\boldsymbol{p}} \sum_{\boldsymbol{q}} \frac{\left(q^{\alpha}-p^{\alpha}\right)(\boldsymbol{p} \times \boldsymbol{q})_{z}}{p^{\alpha} q^{\alpha}} \delta_{\boldsymbol{p}+\boldsymbol{q}-\boldsymbol{k}} \\
& \times[\hat{\theta}(-\boldsymbol{k}, t) \hat{\theta}(\boldsymbol{p}, t) \hat{\theta}(\boldsymbol{q}, t)+\hat{\theta}(\boldsymbol{k}, t) \hat{\theta}(-\boldsymbol{p}, t) \hat{\theta}(-\boldsymbol{q}, t)] \\
\equiv & \sum_{\boldsymbol{p}} \sum_{\boldsymbol{q}} T_{Z}(\boldsymbol{k}, \boldsymbol{p}, \boldsymbol{q}) \equiv \sum_{\boldsymbol{p}} \sum_{\boldsymbol{q}} k^{\alpha} T_{E}(\boldsymbol{k}, \boldsymbol{p}, \boldsymbol{q})
\end{aligned}
$$

where the triad energy transfer function $T_{E}(\boldsymbol{k}, \boldsymbol{p}, \boldsymbol{q})$ is the energy transferred into or out of mode $\boldsymbol{k}$ through interactions with $\boldsymbol{p}$ and $\boldsymbol{q}$, and similarly $T_{Z}(\boldsymbol{k}, \boldsymbol{p}, \boldsymbol{q})=k^{\alpha} T_{E}(\boldsymbol{k}, \boldsymbol{p}, \boldsymbol{q})$ is the enstrophy transferred into or out of mode $\boldsymbol{k}$ through interactions with $\boldsymbol{p}$ and $\boldsymbol{q}$. The triad transfer functions are symmetric in $\boldsymbol{p}$ and $\boldsymbol{q}, T(\boldsymbol{k}, \boldsymbol{p}, \boldsymbol{q})=T(\boldsymbol{k}, \boldsymbol{q}, \boldsymbol{p})$, and vanish if $\boldsymbol{k} \neq \boldsymbol{p}+\boldsymbol{q}$. Energy and enstrophy are conserved in each triad interaction. For general $\alpha$, this is expressed as

$$
\begin{aligned}
T_{E}(\boldsymbol{k}, \boldsymbol{p}, \boldsymbol{q})+T_{E}(\boldsymbol{p}, \boldsymbol{q}, \boldsymbol{k})+T_{E}(\boldsymbol{q}, \boldsymbol{k}, \boldsymbol{p}) & \equiv 0, \\
k^{\alpha} T_{E}(\boldsymbol{k}, \boldsymbol{p}, \boldsymbol{q})+p^{\alpha} T_{E}(\boldsymbol{p}, \boldsymbol{q}, \boldsymbol{k})+q^{\alpha} T_{E}(\boldsymbol{q}, \boldsymbol{k}, \boldsymbol{p}) & \equiv 0 .
\end{aligned}
$$

The triad conservation laws (4.5a) and (4.5b) can be obtained from (4.4) simply by adding the expressions for the appropriate permutations of $\boldsymbol{k}, \boldsymbol{p}$ and $\boldsymbol{q}$ and doing some algebra.

Without loss of generality, let $k \leqslant p \leqslant q$. Following Merilees \& Warn (1975), we define the ratios

$$
X_{E} \equiv-\frac{T_{E}(\boldsymbol{k}, \boldsymbol{p}, \boldsymbol{q})}{T_{E}(\boldsymbol{p}, \boldsymbol{q}, \boldsymbol{k})}=-\frac{q^{\alpha}-p^{\alpha}}{k^{\alpha}-q^{\alpha}}, \quad Y_{E} \equiv-\frac{T_{E}(\boldsymbol{q}, \boldsymbol{k}, \boldsymbol{p})}{T_{E}(\boldsymbol{p}, \boldsymbol{q}, \boldsymbol{k})}=-\frac{p^{\alpha}-k^{\alpha}}{k^{\alpha}-q^{\alpha}},
$$


where $X_{E}$ is the fraction of energy exchanged with larger scales and $Y_{E}$ the fraction exchanged with smaller scales. Note that there is a sign difference between our definition and that of Merilees \& Warn (1975). Owing to the ordering assumption, $X_{E}>0$ and $Y_{E}>0$. This means that $T_{E}(\boldsymbol{k}, \boldsymbol{p}, \boldsymbol{q})$ and $T_{E}(\boldsymbol{q}, \boldsymbol{k}, \boldsymbol{p})$ have the same sign, which is opposite to the sign of $T_{E}(\boldsymbol{p}, \boldsymbol{q}, \boldsymbol{k})$. Hence, transfer is either into the middle wavenumber from the two outlying wavenumbers, or out of the middle wavenumber into the other two. Given a spectrally localized initial distribution, it is natural to assume that the nonlinear interactions spread energy by exciting new modes, in which case one assumes transfers out of the middle wavenumber dominate. However, for a statistically steady spectrum, it is not clear what sense the transfer should have and, in general, transfers both into and out of the middle wavenumber will occur. Moreover, due to the time reversibility of the inviscid equations, for every initial condition in which transfers are one way, there exists an initial condition such that they are the other (Kraichnan 1967).

Since we are interested in triad shapes, not absolute wavenumbers, we express the wavenumbers involved in the triad as fractions of the middle wavenumber, i.e. we set $k=p v, p=p, q=p w$, where $0<v<1,1<w<1+v$. The restriction $w<1+v$ is necessary for the wavevectors to form a closed triangle. The numbers $v$ and $w$ can be used to control the triad shape, since they determine the magnitudes of $k$ and $q$ relative to $p$. Non-localness requires $v \ll 1$, since if $v \approx O(1)$, the requirement that the triad close implies the largest wavevector is similar in size to the other two.

When the wavenumbers are expressed this way, $p$ cancels from the numerator and denominator of (4.6), and we obtain

$$
X_{E}(v, w)=-\frac{w^{\alpha}-1}{v^{\alpha}-w^{\alpha}}, \quad Y_{E}(v, w)=-\frac{1-v^{\alpha}}{v^{\alpha}-w^{\alpha}} .
$$

For enstrophy, we have

$$
X_{Z}(v, w)=-v^{\alpha} \frac{w^{\alpha}-1}{v^{\alpha}-w^{\alpha}}, \quad Y_{Z}(v, w)=-w^{\alpha} \frac{1-v^{\alpha}}{v^{\alpha}-w^{\alpha}} .
$$

Table 1 shows on the left the ratios $X_{E}, Y_{E}, X_{Z}$ and $Y_{Z}$ for the comparatively local triad $0.8 p, p$ and 1.3p $(v=0.8, w=1.3)$. This triad exchanges more (generalized) energy with large than with small scales and more (generalized) enstrophy with smaller than with larger scales for all values of $\alpha$ considered, in both cases the more so for larger $\alpha$. We include results for $\alpha=4$ to show the continued trend of growing energy exchange with larger scales and enstrophy exchange with smaller scales as $\alpha$ increases, noting again that the physical relevance of $\alpha>3$ is questionable since the velocity induced by a point vortex in these models increases with distance (Iwayama \& Watanabe 2010). On the right are the ratios for the non-local triad $0.2 p$, $p$ and $1.1 p(v=0.2, w=1.1)$. Not only does this non-local triad exchange most of its energy with smaller scales, it exchanges almost all of its enstrophy with smaller scales. Combined with the assumption that transfer is out of the middle wavenumber, this indicates simultaneous net downscale transfer of energy and enstrophy as a result of this triad interaction.

It is clear that the triad conservation laws $(4.5 a)$ and $(4.5 b)$ do not preclude net transfer of both energy and enstrophy downscale by a particular triad. Following Merilees \& Warn (1975), we define $S_{E}=X_{E} / Y_{E}$ : when $S_{E}>1$, more energy is exchanged with large scales, and when $S_{E}<1$ more energy is exchanged with small scales for the triad under consideration. Likewise, for enstrophy, we define $S_{Z}=X_{Z} / Y_{Z}$. 
(a)

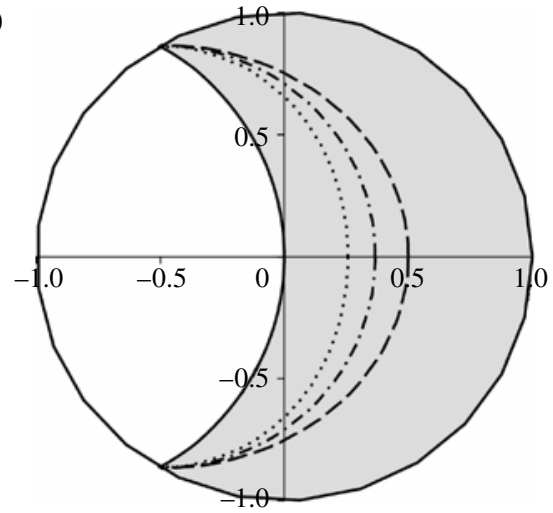

(b)

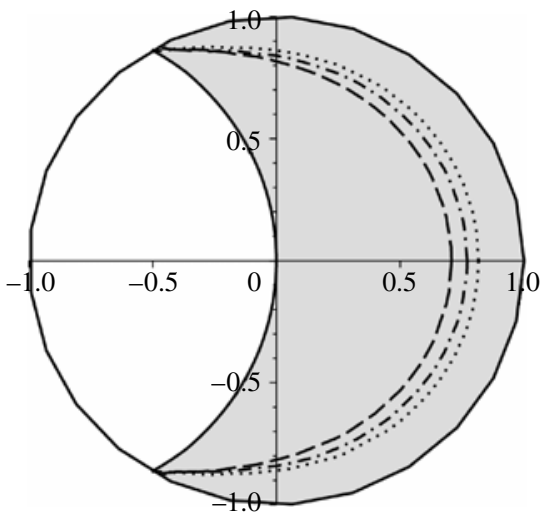

FIGURE 1. The shaded regions, bounded on the left by $\cos \phi=-v / 2$ (arising from $\left.S_{E}, S_{Z} \geqslant 0\right)$, contain triad interactions consistent with $k \leqslant p \leqslant q$. Critical curves $v_{c}(\phi)$ are for (a) $S_{E}$ and (b) $S_{Z}$, for $\alpha=1$ (dashed), $\alpha=2$ (dash-dot) and $\alpha=3$ (dotted). Regions to the left of the critical curves correspond to $S_{E}<1$ and $S_{Z}<1$, i.e. to more exchange with smaller scales. Regions to the right of the critical curves correspond to more exchange with larger scales. Coordinates are polar, with $r=k / p, \phi=\cos ^{-1}(\boldsymbol{k} \cdot \boldsymbol{p} / k p)$.

\begin{tabular}{ccccccccl}
$\alpha$ & 1 & 2 & 3 & 4 & 1 & 2 & 3 & \multicolumn{1}{c}{4} \\
$X_{E}$ & 0.60 & 0.66 & 0.71 & 0.76 & 0.11 & 0.18 & 0.25 & 0.32 \\
$Y_{E}$ & 0.40 & 0.34 & 0.29 & 0.24 & 0.89 & 0.82 & 0.75 & 0.68 \\
$X_{Z}$ & 0.48 & 0.42 & 0.36 & 0.31 & 0.02 & 0.01 & 0.002 & 0.0005 \\
$Y_{Z}$ & 0.52 & 0.58 & 0.64 & 0.69 & 0.98 & 0.99 & 0.998 & 0.9995
\end{tabular}

TABLE 1. Ratios $X_{E}, Y_{E}, X_{Z}$ and $Y_{Z}$ for the local triad shape $v=0.8,1, w=1.3$ (left) and the non-local triad shape $v=0.2,1, w=1.1$ (right).

For the explicit forms of $S_{E}, S_{Z}$ and the critical curves on which $S_{E}=1$ and $S_{Z}=1$, see appendix B.

Plots of the critical curves for $S_{E}$ appear in figure 1(a), and for $S_{Z}$ in figure $1(b)$. As $\alpha$ increases, the region for which $S_{E}>1$ grows, i.e. more triads exchange most of their energy with large rather than with small scales. Conversely, as $\alpha$ increases, more triads exchange the bulk of their enstrophy with small scales, which is reflected in the growing region for which $S_{Z}<1$. Further, as reflected in table 1 , the fractions of energy and enstrophy exchanged with large and small scales, respectively, increase with $\alpha$.

In figure 2, the critical $S_{E}$ and $S_{Z}$ curves appear on the same plot for each of $\alpha=1,2,3$. As $\alpha$ increases, so does the percentage of triads that exchange both more energy with larger scales and more enstrophy with smaller scales. These triads fall in the wedge between the dotted and dashed lines, which increases in area as $\alpha$ increases. This might be taken to support the expectation of an inverse energy cascade and a direct enstrophy cascade, both strengthening as $\alpha$ increases. However, to make predictions about turbulent fluxes based on the above geometrical arguments, one would have to make assumptions both about how dynamically active various triads are and about the sense of the transfer. Specifically, to predict an inverse energy cascade and a direct enstrophy cascade, one would have to assume that neither very 
(a)

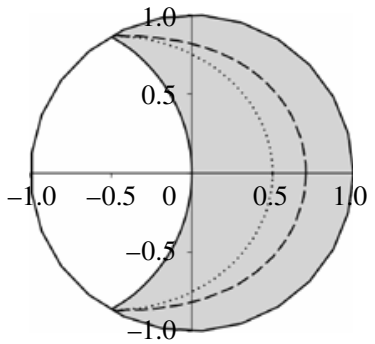

(b)

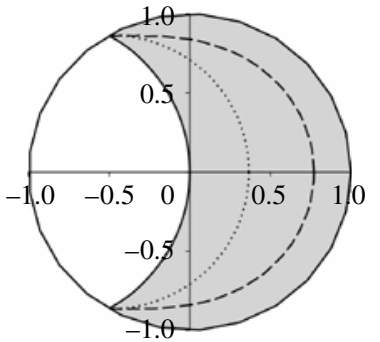

$(c)$

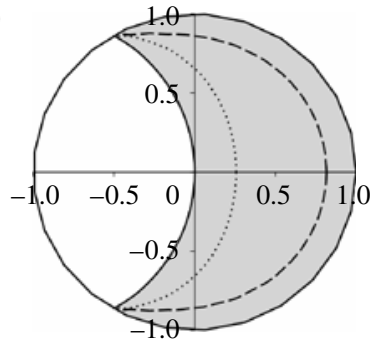

FIgURE 2. Critical $S_{E}$ (dotted) and $S_{Z}$ (dashed) curves for $(a) \alpha=1,(b) \alpha=2$ and $(c) \alpha=3$. Here $S_{E}>1$ and $S_{Z}<1$ in the region between the dotted and dashed lines, while $S_{E}<1$ and $S_{Z}<1$ in the region to the left of the dotted line and $S_{E}>1$ and $S_{Z}>1$ to the right of the dashed line. Coordinates are as in figure 1 .

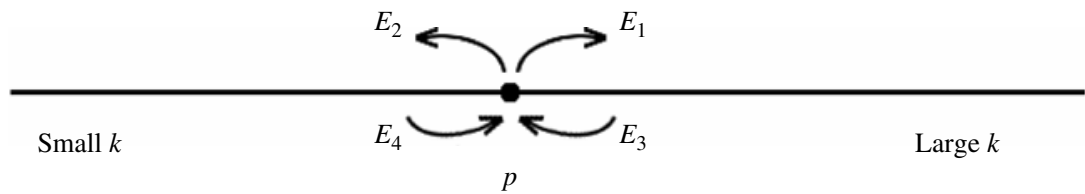

FIGURE 3. Energy transferred into and out of wavevector $\boldsymbol{p}$ by two different triads. See the text for details.

non-local nor very local triads dominated the fluxes, and that transfer was out of the middle wavenumber. Such assumptions may be valid in certain inertial ranges, but not in others. In reality, geometrical arguments provide no information about dynamical activity, and ad hoc assumptions about the importance of various triads and the sense of the transfer in statistically steady inertial ranges lack adequate motivation.

Fjørtoft arguments do not, in fact, preclude turbulent transfers quite counter to common intuition. For example, one can find transfers involving two allowed triads sharing a common middle wavenumber such that the net energy transfer is downscale and the net enstrophy transfer is zero. Consider two triads engaged in the transfers depicted in figure 3, where the central wavenumber is indicated with a dot, the sense of the transfer with the arrows and $E_{1}, E_{2}, E_{3}, E_{4}>0$. We enforce that the interactions leave the energy and enstrophy in $\boldsymbol{p}$ unchanged, as would be the case in an equilibrated inertial range, by requiring

$$
E_{1}+E_{2}=E_{3}+E_{4}, \quad Z_{1}+Z_{2}=Z_{3}+Z_{4},
$$

where $Z_{1}$ is the enstrophy associated with $E_{1}$, etc. Now, let the two triads in question be $k<p<q$ and $k^{\prime}<p<q^{\prime}$. Then

$$
Z_{1}=q^{\alpha} E_{1}>p^{\alpha} E_{1}, \quad Z_{2}=k^{\alpha} E_{2}<p^{\alpha} E_{2} .
$$

Similarly, we have

$$
Z_{3}=\left(q^{\prime}\right)^{\alpha} E_{3}>p^{\alpha} E_{3}, \quad Z_{4}=\left(k^{\prime}\right)^{\alpha} E_{4}<p^{\alpha} E_{4} .
$$

We show by explicit construction that the hypothesized situation is possible. Let $E_{1}=1.5, E_{2}=3, E_{3}=1.4, E_{4}=3.1$ and $Z_{1}=Z_{2}=Z_{3}=Z_{4}=2$. Then (4.9) is satisfied, 
and

$$
k^{\alpha}=\frac{Z_{2}}{E_{2}}=\frac{2}{3}, \quad p^{\alpha}=\frac{Z_{1}+Z_{2}}{E_{1}+E_{2}}=\frac{8}{9}, \quad q^{\alpha}=\frac{Z_{1}}{E_{1}}=\frac{4}{3} .
$$

Hence, both the ordering assumption and conditions (4.10) are also satisfied. Recalling that $q<k+p$ for the triad to close, one can verify that this triad closes for all $\alpha>0$ by solving

$$
\left(\frac{4}{3}\right)^{1 / \alpha_{c}}-\left(\frac{2}{3}\right)^{1 / \alpha_{c}}-\left(\frac{8}{9}\right)^{1 / \alpha_{c}}=0
$$

numerically for $\alpha_{c}$, which yields $\alpha_{c}=-0.66$.

Similarly,

$$
\left(k^{\prime}\right)^{\alpha}=\frac{Z_{4}}{E_{4}}=\frac{20}{31}, \quad p^{\alpha}=\frac{Z_{3}+Z_{4}}{E_{3}+E_{4}}=\frac{8}{9}, \quad\left(q^{\prime}\right)^{\alpha}=\frac{Z_{3}}{E_{3}}=\frac{10}{7},
$$

so the ordering assumption for the second triad and the conditions (4.11) are satisfied. One can verify that this triad closes for $\alpha>0.90$, and so for SQG, Navier-Stokes and RSF, by solving the equation equivalent to (4.13).

Hence, these two triads and associated transfers are allowed by both (4.9) and the ordering assumption, and their net effect is downscale energy transfer, since $E_{1}+E_{4}=$ $4.6>4.4=E_{2}+E_{3}$, and zero net enstrophy transfer, since $Z_{1}+Z_{4}=4=Z_{2}+Z_{3}$. Such transfers are consistent with a downscale energy-cascading inertial range. We would not expect such an inertial range to be physically realizable in a statistically steady state: it would require both zero net enstrophy flux through the inertial range and enstrophy to be dissipated at a rate $k_{d}^{\alpha} \varepsilon$ (where $k_{d}$ is a dissipation wavenumber and $\varepsilon$ the energy flux), requirements that are contradictory. The point we wish to make is that such an inertial range is not in contravention of triad conservation of energy and enstrophy, and is allowed a priori by Fjørtoft arguments.

\section{EDQNM closure and similarity solutions for generalized 2D fluids}

To gain further insight into the sense of the transfers and the directions of the fluxes in generalized 2D turbulence, we now add dynamics in first approximation using the EDQNM approximation. To probe the validity of KLB inertial range theory for the inverse cascade, we make a similarity assumption and seek self-similar inertial range solutions. The similarity assumption also allows us to write the flux in such a way that we can study the contributions from triads of various shapes.

\subsection{EDQNM closure}

The EDQNM approximation is a method of closing the turbulent moment hierarchy that involves making assumptions of limited Gaussianity. It is a variant of the DIA (Kraichnan 1958a,b, 1959), which corresponds to the lowest order of classical renormalized perturbation theory (Martin et al. 1973). The essential assumption behind the DIA is that of maximal randomness: any correlations are assumed to develop via nonlinear interactions and not to arise from ordered initial conditions or forcing. As a statistical closure, the EDQNM describes the average behaviour of a turbulent ensemble. The dynamical variable of the theory is no longer the velocity or vorticity field, but rather the energy spectrum itself. 
We take the infinite system limit, $L \rightarrow \infty$, in which $V \sum_{p} \rightarrow \int \mathrm{d} \boldsymbol{p}$, where $V=(2 \pi / L)^{2}$. The ensemble-averaged second and third moments are

$$
\begin{aligned}
\Theta(\boldsymbol{k}) & =\lim _{V \rightarrow 0} V^{-1}\langle\hat{\theta}(\boldsymbol{k}) \hat{\theta}(-\boldsymbol{k})\rangle, \\
\mathscr{R}(\boldsymbol{k}, \boldsymbol{p}, \boldsymbol{q}) & =\lim _{V \rightarrow 0} V^{-2}\langle\hat{\theta}(\boldsymbol{k}) \hat{\theta}(\boldsymbol{p}) \hat{\theta}(\boldsymbol{q})\rangle .
\end{aligned}
$$

After ensemble averaging, and including viscosity, equation (4.4) can be expressed as

$$
\begin{aligned}
\left(\frac{\partial}{\partial t}+2 v k^{2}\right) \Theta(\boldsymbol{k})= & \frac{1}{2} \int \mathrm{d} \boldsymbol{p} \mathrm{d} \boldsymbol{q} \frac{\left(q^{\alpha}-p^{\alpha}\right)(\boldsymbol{p} \times \boldsymbol{q})_{z}}{p^{\alpha} q^{\alpha}} \delta^{2}(\boldsymbol{p}+\boldsymbol{q}-\boldsymbol{k}) \\
& \times[\mathscr{R}(-\boldsymbol{k}, \boldsymbol{p}, \boldsymbol{q})+\mathscr{R}(\boldsymbol{k},-\boldsymbol{p},-\boldsymbol{q})] .
\end{aligned}
$$

To obtain a closed equation for the energy spectrum, we must express $\mathscr{R}$ in terms of $\Theta$. Following the standard EDQNM procedure (see appendix $C$ for details), we obtain

$$
\begin{aligned}
T_{E}(k)= & \frac{1}{\pi} \int_{0}^{\infty} \mathrm{d} p \int_{|k-p|}^{k+p} \mathrm{~d} q D_{k p q} \frac{k^{2}}{p q} \\
& \times\left[2 a_{k p q} k \mathscr{E}(p) \mathscr{E}(q)-b_{k p q} p \mathscr{E}(k) \mathscr{E}(q)-b_{k q p} q \mathscr{E}(k) \mathscr{E}(p)\right] \\
= & \frac{1}{2} \int_{0}^{\infty} \mathrm{d} p \int_{|k-p|}^{k+p} \mathrm{~d} q T_{E}(k, p, q),
\end{aligned}
$$

where we have assumed isotropy and used the relation

$$
\mathscr{E}(k)=\pi k^{1-\alpha} \Theta(k) .
$$

Here

$$
2 a_{k p q}=b_{k p q}+b_{k q p}, \quad b_{k p q}=2 \frac{\left(k^{\alpha}-q^{\alpha}\right)\left(p^{\alpha}-q^{\alpha}\right)}{k^{\alpha+2}(p q)^{\alpha-2}}\left(1-x^{2}\right)^{1 / 2},
$$

where $x$ is the cosine of the angle opposite $\boldsymbol{k}$ in the triad $\boldsymbol{k}, \boldsymbol{p}, \boldsymbol{q}$, i.e.

$$
x=\frac{\boldsymbol{p} \cdot \boldsymbol{q}}{p q} .
$$

A Markovianized eddy-damping function, $D_{k p q}$, replaces viscous damping. The eddydamping function is an effective memory time for the triad $\boldsymbol{k}, \boldsymbol{p}, \boldsymbol{q}$, and represents turbulent scrambling of the triple correlation function. Replacement of molecular viscous effects with eddy damping is necessary to avoid excessive growth of the third moment and negative energy spectra (Orszag 1970). We use the phenomenological expression

$$
D_{k p q}=\left\{\mu\left[\tau(k)^{-1}+\tau(p)^{-1}+\tau(q)^{-1}\right]\right\}^{-1},
$$

where $\tau$ is the eddy turnover time defined by (2.4) and $\mu$ is a dimensionless constant that can be determined by requiring agreement between the TFM and DIA in a particular case (Kraichnan 1971a,b).

The coefficients satisfy

$$
\begin{aligned}
\frac{k^{2}}{p q} b_{k p q} & =\frac{p^{2}}{k q} b_{p k q}, \\
\frac{k^{\alpha+2}}{p q}\left[b_{k p q}+b_{k q p}\right] & =\frac{p^{\alpha+2}}{k q} b_{p k q}+\frac{q^{\alpha+2}}{k p} b_{q k p} .
\end{aligned}
$$


As a result, the closure (5.4) satisfies the triad conservation laws (4.5a) and (4.5b).

Setting $\alpha=2$ in (5.1), (5.4) and (5.6), we recover the standard result for $2 \mathrm{D}$ Navier-Stokes turbulence (Kraichnan 1971b; Leith 1971). To obtain the closure for the enstrophy inertial range, one simply multiplies (5.4) by $k^{\alpha}$ and uses the appropriate energy spectrum and eddy turnover time. The coefficients $a_{k p q}$ and $b_{k p q}$ do not change, since they are determined by triad geometry.

\subsection{Self-similar inertial range solutions}

We now seek self-similar inertial range solutions by assuming a power-law scaling for the energy spectrum and making a similarity assumption for the triad transfer function. First, we rewrite the flux, starting with the evolution equation for the energy spectrum, where we have neglected viscosity because we are interested in inertial range dynamics far away from the dissipation scale,

$$
(\partial / \partial t) \mathscr{E}(k)=T_{E}(k)=\frac{1}{2} \int_{0}^{\infty} \int_{0}^{\infty} T_{E}(k, p, q) \mathrm{d} p \mathrm{~d} q .
$$

No ordering of $\boldsymbol{k}, \boldsymbol{p}, \boldsymbol{q}$ is assumed at this point, and certainly not the ordering in $\S 4$. The flux of energy per unit mass from wavenumbers less than $k$ to wavenumbers greater than $k$ is

$$
\pi_{E}(k)=\int_{k}^{\infty} T_{E}\left(k^{\prime}\right) \mathrm{d} k^{\prime}=-\int_{0}^{k} T_{E}\left(k^{\prime}\right) \mathrm{d} k^{\prime}
$$

by virtue of

$$
\int_{0}^{\infty} T_{E}\left(k^{\prime}\right) \mathrm{d} k^{\prime}=0
$$

i.e. the fact that nonlinear interactions merely transfer generalized energy between wavenumbers, rather than changing the total amount.

Using (4.5a), the flux past $k$ can thus be decomposed into gains at $k^{\prime}>k$ due to interactions with $p, q<k$ and losses at $k^{\prime}<k$ due to interactions with $p, q>k$ :

$$
\begin{aligned}
\pi_{E}(k)= & \frac{1}{2} \int_{k}^{\infty} \mathrm{d} k^{\prime} \int_{0}^{k} \int_{0}^{k} T_{E}\left(k^{\prime}, p, q\right) \mathrm{d} p \mathrm{~d} q \\
& -\frac{1}{2} \int_{0}^{k} \mathrm{~d} k^{\prime} \int_{k}^{\infty} \int_{k}^{\infty} T_{E}\left(k^{\prime}, p, q\right) \mathrm{d} p \mathrm{~d} q .
\end{aligned}
$$

See appendix D for a detailed derivation of this result. Downscale flux is positive.

We will refer to triads with $p, q<k<k^{\prime}$ as type a triads, and triads with $k^{\prime}<k<p, q$ as type $b$ triads. The corresponding flux of enstrophy is

$$
\begin{aligned}
\pi_{Z}(k)= & \frac{1}{2} \int_{k}^{\infty}\left(k^{\prime}\right)^{\alpha} \mathrm{d} k^{\prime} \int_{0}^{k} \int_{0}^{k} T_{E}\left(k^{\prime}, p, q\right) \mathrm{d} p \mathrm{~d} q \\
& -\frac{1}{2} \int_{0}^{k}\left(k^{\prime}\right)^{\alpha} \mathrm{d} k^{\prime} \int_{k}^{\infty} \int_{k}^{\infty} T_{E}\left(k^{\prime}, p, q\right) \mathrm{d} p \mathrm{~d} q .
\end{aligned}
$$

Following Kraichnan (1967), we assume similarity laws for the energy spectrum and triple moment. For general $\alpha$, the triple moment has the same dimensions as $[\mathscr{E}(k)]^{3 / 2} k^{(1-\alpha) / 2}$, so we assume the following scalings:

$$
\begin{aligned}
\mathscr{E}(a k) / \mathscr{E}(k) & =a^{-n} \\
T_{E}(a k, a p, a q) / T_{E}(k, p, q) & =a^{(1-3 n-\alpha) / 2} .
\end{aligned}
$$


Because $T_{E}(k, p, q)$ is symmetric in $p$ and $q, \int_{0}^{k} \mathrm{~d} p \int_{0}^{k} \mathrm{~d} q$ is equivalent to $2 \int_{0}^{k} \mathrm{~d} p \int_{0}^{p} \mathrm{~d} q$ in the first integral of (5.14), while $\int_{k}^{\infty} \mathrm{d} p \int_{k}^{\infty} \mathrm{d} q$ is equivalent to $2 \int_{k}^{\infty} \mathrm{d} p \int_{p}^{\infty} \mathrm{d} q$ in the second integral. These choices of integration regions reflect the wavenumber orderings we have chosen in the two terms $\left(q \leqslant p \leqslant k^{\prime}\right.$ and $k^{\prime} \leqslant p \leqslant q$, respectively).

Our next step is to express the wavenumber magnitudes in terms of $k$. We will work with the two terms in (5.14) separately, since they require different scalings. In the first term, the triads (type a) are such that $q \leqslant p \leqslant k \leqslant k^{\prime}$. We set

$$
p=k / u, \quad q=p v, \quad k^{\prime}=p w .
$$

Since $k$ is fixed, $u, v$ and $w$ are regarded as variable in these expressions. For fixed $k, u$ determines the triad size, as measured by the magnitude $p$. As before, $v$ and $w$ determine the triad shape by controlling the relative magnitudes of the other two wavenumbers. By choosing $u, v$ and $w$ appropriately, we can generate differently sized triads that all have the same shape, i.e. the same interior angles. We will then express the flux as integrals over the ratios $u=k / p, v=q / p$ and $w=k^{\prime} / p$, i.e. as integrals over triangle size $u$, and triangle shapes, parameterized by $v$ and $w$, rather than integrals over wavenumbers.

We must now obtain the appropriate limits for the new integration variables. By assumption, $u \geqslant 1$, since $p \leqslant k$, and $v \leqslant 1$, since $q \leqslant p$. Then (5.17) implies $w \geqslant u$, since $k^{\prime} \geqslant k \geqslant p$, and $v \geqslant 0$, since $q \geqslant 0$. The integration ranges are thus $1 \leqslant u \leqslant w$, $1 \leqslant w \leqslant 1+v$ and $0 \leqslant v \leqslant 1$.

In the second integral of (5.14), the triads are such that $k^{\prime} \leqslant k \leqslant p \leqslant q$ (type $\mathrm{b}$ triads). The appropriate scalings are

$$
p=k / u, \quad k^{\prime}=p v, \quad q=p w,
$$

and the integration ranges are $v \leqslant u \leqslant 1,0 \leqslant v \leqslant 1$, and $1 \leqslant w \leqslant 1+v$.

Re-expressing (5.14) in terms of $k, u, v$ and $w$, we obtain

$$
\begin{aligned}
\pi_{E}(k)= & \int_{0}^{1} \mathrm{~d} v \int_{1}^{1+v} \mathrm{~d} w \\
& \times\left[\int_{1}^{w} \mathrm{~d} u p^{4} k^{-1} T_{E}(p w, p, p v)-\int_{v}^{1} \mathrm{~d} u p^{4} k^{-1} T_{E}(p v, p, p w)\right] .
\end{aligned}
$$

Inverting the first expression in (4.6) and assuming isotropy, we obtain

$$
T_{E}(p, q, k) / T_{E}(k, p, q)=\left(q^{\alpha}-k^{\alpha}\right) /\left(p^{\alpha}-q^{\alpha}\right) .
$$

We now re-express $T_{E}(p w, p, p v)$ using the similarity assumption (5.16b) and (5.20) with $p w, p, p v$ in place of $k, p, q$, respectively,

$$
T_{E}(p w, p, p v)=T_{E}(1, v, w) \frac{1-v^{\alpha}}{v^{\alpha}-w^{\alpha}} p^{(1-3 n-\alpha) / 2} .
$$

For the second term in (5.19), we use symmetry of the triad transfer function in its second two arguments to write $T_{E}(p v, p, p w)=T_{E}(p v, p w, p)$. Using (5.16b) and (5.20) with $p, p v, p w$ in place of $k, p, q$ then gives

$$
T_{E}(p v, p, p w)=T_{E}(1, v, w) \frac{w^{\alpha}-1}{v^{\alpha}-w^{\alpha}} p^{(1-3 n-\alpha) / 2} .
$$


Substituting these into $\pi_{E}(k)$, we obtain

$$
\pi_{E}(k)=k^{(7-3 n-\alpha) / 2} \int_{0}^{1} \mathrm{~d} v \int_{1}^{1+v} \mathrm{~d} w W_{E}^{\alpha}(v, w, n) T_{E}(1, v, w)
$$

where $W_{E}^{\alpha}(v, w, n)$, the triad weight, is given by

$$
\begin{aligned}
W_{E}^{\alpha}(v, w, n)= & -\left(w^{\alpha}-v^{\alpha}\right)^{-1} \\
& \times\left[\left(1-v^{\alpha}\right) \int_{1}^{w} u^{(3 n+\alpha-9) / 2} \mathrm{~d} u-\left(w^{\alpha}-1\right) \int_{v}^{1} u^{(3 n+\alpha-9) / 2} \mathrm{~d} u\right] .
\end{aligned}
$$

Note that for $n=(7-\alpha) / 3$, we obtain a $k$-independent energy flux, in which case

$$
W_{E}^{\alpha}[v, w,(7-\alpha) / 3]=-\left(w^{\alpha}-v^{\alpha}\right)^{-1}\left[\left(1-v^{\alpha}\right) \ln w+\left(w^{\alpha}-1\right) \ln v\right] .
$$

The power law $n=(7-\alpha) / 3$ is that predicted by a Kolmogorov locality assumption for the energy spectrum in the energy inertial range. (See $(2.3 a)$.) This is to be expected, since assuming a similarity scaling for the triple correlation function amounts to assuming that forcing and dissipation scales do not explicitly enter the dynamics, and that all three wavenumbers lie in the same inertial range. In the limit of infinite inertial ranges, the similarity scaling requires transfers to be local in the sense that the flux is not dominated by contributions from $v \rightarrow 0$. If the flux is dominated by contributions from $v \rightarrow 0$, the infrared cutoff will determine the dynamics no matter how large the inertial range. On the other hand, the flux could still be dominated by very long, thin triads, i.e. contributions from $v \ll 1$, without the similarity theory failing.

Similar manipulations lead to the following expression for the enstrophy flux:

$$
\pi_{Z}(k)=k^{(7-3 n+\alpha) / 2} \int_{0}^{1} \mathrm{~d} v \int_{1}^{1+v} \mathrm{~d} w W_{Z}^{\alpha}(v, w, n) T_{E}(1, v, w)
$$

with

$$
\begin{aligned}
W_{Z}^{\alpha}(v, w, n)= & -\left(w^{\alpha}-v^{\alpha}\right)^{-1} \\
& \times\left[\left(1-v^{\alpha}\right) w^{\alpha} \int_{1}^{w} u^{(3 n-\alpha-9) / 2} \mathrm{~d} u-\left(w^{\alpha}-1\right) v^{\alpha} \int_{v}^{1} u^{(3 n-\alpha-9) / 2} \mathrm{~d} u\right] .
\end{aligned}
$$

For a $k$-independent flux, we need $n=(7+\alpha) / 3$, which is the dimensionally predicted power law $(2.3 b)$ for the energy spectrum in the enstrophy inertial range. The triad weight is then

$$
W_{Z}^{\alpha}[v, w,(7+\alpha) / 3]=-\left(w^{\alpha}-v^{\alpha}\right)^{-1}\left[\left(1-v^{\alpha}\right) w^{\alpha} \ln w+\left(w^{\alpha}-1\right) v^{\alpha} \ln v\right] .
$$

Substituting $n=(7+\alpha) / 3$ into (5.24), one finds that $W_{E}[v, w,(7+\alpha) / 3]=0$, i.e. that the energy flux vanishes in the self-similar enstrophy-cascading inertial range. Similarly, substituting $n=(7-\alpha) / 3$ into (5.27), one finds that $W_{Z}[v, w,(7-\alpha) / 3]=0$, so the enstrophy flux vanishes in the self-similar energy-cascading inertial range.

The factors $W_{E}^{\alpha}(v, w, n)$ and $W_{Z}^{\alpha}(v, w, n)$ partially determine how much various triangle shapes, integrated over triangle size $u$, contribute to the fluxes (Kraichnan 1967). The first terms of (5.24) and (5.27) are the weights for the contribution of triads $q \leqslant p \leqslant k \leqslant k^{\prime}$ (type a triads) to the flux, while the second terms weight the contribution of triads with $k^{\prime} \leqslant k \leqslant p \leqslant q$ (type $\mathrm{b}$ triads). The terms $X_{E}(v, w)$ and $Y_{E}(v, w)$, as defined in the Fjørtoft analysis (4.7), appear as factors in the first and second terms of (5.25), multiplying the integrations over triad size. Hence, the 
percentage of energy exchanged with smaller scales in a given triad interaction appears as a factor in the weight for type a triads, while the percentage of energy exchanged with larger scales appears as a factor in the weight for type b triads. The weight function differs from the Fjørtoft analysis in the integration over triad size, which appears by virtue of the similarity assumption for the triad transfer function (an assumption not made in the Fjørtoft analysis). We will study the total fluxes and the contributions from each triad type separately. As we will see, the EDQNM closure predicts that the flux from type a triads is directed oppositely to that from the type $b$ triads.

We seek solutions with $k$-independent flux, eliminating the factor of $k^{(7-3 n-\alpha) / 2}$ by setting $n=(7-\alpha) / 3$. Substituting (5.4) for $T_{E}(1, v, w)$ in (5.23), and using (2.3a) and (5.8) yields

$$
\begin{aligned}
\pi_{E}(k)= & \frac{2 C^{3 / 2} \varepsilon}{\pi \mu} \int_{0}^{1} \mathrm{~d} v \int_{1}^{1+v} \mathrm{~d} w W_{E}^{\alpha}[v, w,(7-\alpha) / 3] \tilde{D}_{1 v w}^{E} \\
& \times \frac{1}{v w}\left[2 a_{1 v w}(v w)^{-(7-\alpha) / 3}-b_{1 v w} v w^{-(7-\alpha) / 3}-b_{1 w v} w v^{-(7-\alpha) / 3}\right] \\
\equiv & \varepsilon C^{3 / 2} \mu^{-1} \int_{0}^{1} \frac{\mathrm{d} v}{v} \int_{1}^{1+v} \mathrm{~d} w Q_{E}(v, w) \\
\equiv & \varepsilon C^{3 / 2} \mu^{-1} \int_{0}^{1} \frac{\mathrm{d} v}{v} Q_{E}(v),
\end{aligned}
$$

where

$$
\tilde{D}_{1 v w}^{E}=\left[1+v^{(4-\alpha) / 3}+w^{(4-\alpha) / 3}\right]^{-1}
$$

and

$$
C^{3 / 2} \mu^{-1} \int_{0}^{1} \frac{\mathrm{d} v}{v} Q_{E}(v)= \pm 1 .
$$

Here $C$ is an $\alpha$-dependent positive dimensionless constant, the Kolmogorov-Kraichnan constant appearing in (2.3a), and $\varepsilon$, which is taken to be positive, has units of energy flux and is $k$-independent. The function $Q_{E}(v)$ scales with $v$ the same way as the flux in the limit $v \rightarrow 0$.

The fraction of inertial range energy transfer due to triads in which the ratio of the smallest to middle wavenumber is less than $v$ is given by

$$
F_{E}(v)=C^{3 / 2} \mu^{-1} \int_{0}^{v} \frac{\mathrm{d} s}{s} Q_{E}(s)=\frac{\int_{0}^{v}(\mathrm{~d} s / s) Q_{E}(s)}{\int_{0}^{1}(\mathrm{~d} s / s) Q_{E}(s)} \operatorname{sgn}\left[\pi_{E}(k)\right] .
$$

We will call $F_{E}(v)$ the flux fraction. Note that this is a signed quantity by virtue of (5.31).

Similarly, for the enstrophy inertial range, we obtain

$$
\begin{aligned}
\pi_{Z}(k)= & \frac{2 C^{3 / 2} \eta}{\pi \mu} \int_{0}^{1} \mathrm{~d} v \int_{1}^{1+v} \mathrm{~d} w W_{Z}^{\alpha}[v, w,(7+\alpha) / 3] \tilde{D}_{1 v w}^{Z} \\
& \times \frac{1}{v w}\left[2 a_{1 v w}(v w)^{-(7+\alpha) / 3}-b_{1 v w} v w^{-(7+\alpha) / 3}-b_{1 w v} w v^{-(7+\alpha) / 3}\right]
\end{aligned}
$$




$$
\begin{aligned}
& \equiv \eta C^{\prime 3 / 2} \mu^{-1} \int_{0}^{1} \frac{\mathrm{d} v}{v} \int_{1}^{1+v} \mathrm{~d} w Q_{Z}(v, w) \\
& \equiv \eta C^{\prime 3 / 2} \mu^{-1} \int_{0}^{1} \frac{\mathrm{d} v}{v} Q_{Z}(v),
\end{aligned}
$$

where

$$
\tilde{D}_{1 v w}^{Z}=\left[1+v^{(4-2 \alpha) / 3}+w^{(4-2 \alpha) / 3}\right]^{-1},
$$

$C^{\prime}$ is the dimensionless Kolmogorov-Kraichnan constant appearing in (2.3b) and $\eta$ has units of enstrophy flux, which is again $k$-independent due to the choice $n=(7+\alpha) / 3$. The fraction of the flux due to triads in which the ratio of the smallest to middle wavenumbers is less than $v$ is

$$
F_{Z}(v)=C^{\prime 3 / 2} \mu^{-1} \int_{0}^{v} \frac{\mathrm{d} s}{s} Q_{Z}(s)=\frac{\int_{0}^{v}(\mathrm{~d} s / s) Q_{Z}(s)}{\int_{0}^{1}(\mathrm{~d} s / s) Q_{Z}(s)} \operatorname{sgn}\left[\pi_{Z}(k)\right] .
$$

\subsection{Behaviour of $Q_{E}(v)$ and $F_{E}(v)$ as $v \rightarrow 0$}

The flux fraction (5.32) may diverge for certain $\alpha$ in the limit $v \rightarrow 0$, indicating that triads with $v \rightarrow 0$ dominate the flux, and signalling complete breakdown of locality. In this case, assuming a self-similar inertial range where (5.16a) and (5.16b) hold is not valid, so studying the behaviour of $F_{E}(v)$ in this limit allows us to check the consistency of our assumptions. Note that, in this limit, $F_{E}(v)$ and $Q_{E}(v)$ scale with $v$ the same way. To study this possible divergence and its dependence on $\alpha$, for $v \ll 1$, we approximate $Q_{E}(v)$ with a middle Riemann sum. (We must take the middle sum because the integrand vanishes for $w=1$ and $w=1+v$.) That is, setting $w=1+v / 2$, $\mathrm{d} w=v$, we study

$$
\begin{aligned}
Q_{E}(v) \propto & \frac{v}{w} W_{E}^{\alpha}[v, w,(7-\alpha) / 3] \tilde{D}_{1 v w}^{E} \\
& \times\left[2 a_{1 v w}(v w)^{-(7-\alpha) / 3}-b_{1 v w} v w^{-(7-\alpha) / 3}-b_{1 w v} w v^{-(7-\alpha) / 3}\right],
\end{aligned}
$$

and find the leading-order term as $v \rightarrow 0$. The result is

$$
\begin{aligned}
Q_{E}(v)=O\left[v^{2} \ln (1 / v)\right] & (\alpha=1, v \ll 1), \\
Q_{E}(v)=O\left[v^{4 / 3} \ln (1 / v)\right] & (\alpha=2, v \ll 1), \\
Q_{E}(v)=O\left[v^{2 / 3} \ln (1 / v)\right] & (\alpha=3, v \ll 1), \\
Q_{E}(v)=O[\ln (1 / v)] & (\alpha=4, v \ll 1) .
\end{aligned}
$$

The result for $\alpha=2$ agrees with that found by Kraichnan (1971b). We see that $Q_{E}(v)$, and therefore also the EDQNM energy flux, diverges as $v \rightarrow 0$ for $\alpha \geqslant 4$. This means that for $\alpha \geqslant 4$ triads with $v \rightarrow 0$ dominate the flux, transfers are truly and unavoidably non-local, and seeking a self-similar inertial range where (5.16a) and (5.16b) hold is inconsistent. An infinite flux also means the model we are considering, an EDQNM closure combined with similarity scaling, has clearly become unphysical.

The divergence of the energy flux for $\alpha \geqslant 4$ is consistent with the eddy turnover time (2.4) becoming $k$-independent in the energy inertial range for $\alpha=4$ and increasing with $k$ for $\alpha>4$. Both of these results seem to suggest that KLB energycascading inertial ranges with the similarity spectrum $(2.3 a)$ should be realizable 


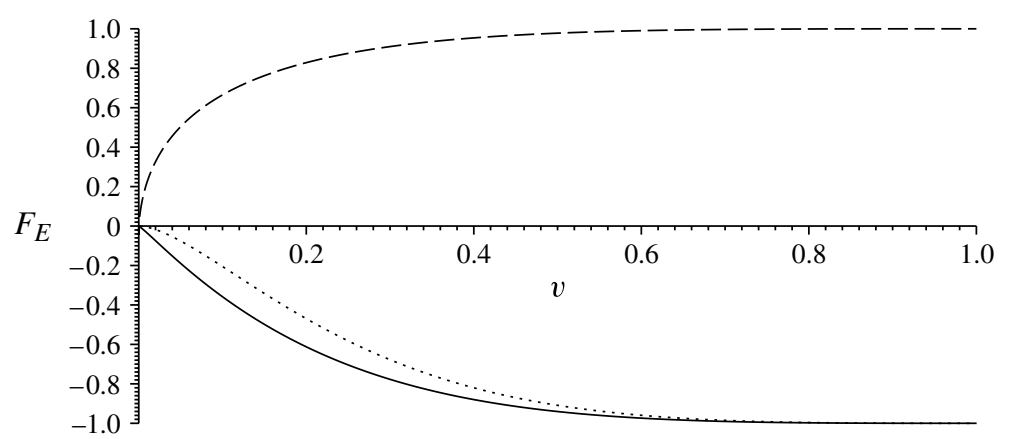

FIGURE 4. Flux fraction for $\alpha=1$ (dotted line), $\alpha=2$ (solid line) and $\alpha=3$ (dashed line).

for $\alpha<4$. On the other hand, we might predict that the energy cascade becomes non-local for $\alpha \geqslant 4$, and not expect similarity theory to adequately describe such models.

\subsection{Behaviour of $Q_{Z}(v)$ and $F_{Z}(v)$ as $v \rightarrow 0$}

Repeating the above analysis for $Q_{Z}(v)$, we obtain

$$
\begin{aligned}
Q_{Z}(v)=O\left[v^{7 / 3} \ln (1 / v)\right] & (\alpha=1, v \ll 1), \\
Q_{Z}(v)=O(1) & (\alpha=2, v \ll 1), \\
Q_{Z}(v)=O\left(v^{-2 / 3}\right) & (\alpha=3, v \ll 1), \\
Q_{Z}(v)=O\left(v^{-4 / 3}\right) & (\alpha=4, v \ll 1) .
\end{aligned}
$$

The enstrophy flux diverges for $\alpha \geqslant 2$, indicating that transfers become non-local and assuming a self-similar inertial range where (5.16a) and (5.16b) hold is inconsistent for $\alpha \geqslant 2$. This is consistent with the behaviour of the eddy turnover time, which becomes $k$-independent at $\alpha=2$ and increases with $k$ for $\alpha>2$. It is also in agreement with well-established results: Kraichnan $(1971 b)$ predicted the need for a logarithmic correction to the enstrophy spectrum for $\alpha=2$. Numerical simulation shows that the dynamics in the generalized enstrophy cascading range is non-local for $\alpha>2$, with the spectrum shallowing to that of a passive tracer (Watanabe \& Iwayama 2004).

\section{Non-locality and flux in the energy inertial range}

Figure 4 shows the energy flux fraction $F_{E}(v)$ for $\alpha \in\{1,2,3\}$. For $\alpha \in\{1,2\}$, the flux fraction is negative, indicating net upscale transfer, i.e. the similarity ranges are associated with inverse energy cascades. We denote the values of $v$ at which the flux fraction reaches $80 \%$ as $v_{80 \%}$. For $\alpha=1, v_{80 \%} \approx 0.38$, while for $\alpha=2, v_{80 \%} \approx 0.32$. As expected, non-local triads make a larger contribution to the flux as $\alpha$ increases from 1 to 2 . For $\alpha=3$, the flux is positive; surprisingly, the KLB self-similar inertial range solution for this value of $\alpha$ is associated with a downscale energy cascade. As stated earlier, we do not expect such a downscale energy cascade to be physically realizable, as it would require simultaneous zero enstrophy flux through the inertial range and non-zero enstrophy dissipation (at a rate $k_{d}^{\alpha} \varepsilon$, where $k_{d}$ is a characteristic dissipation wavenumber). We further investigate this downscale energy flux and its implications for 2D energy cascades below. For $\alpha=3$, the flux is also dominated by considerably more non-local triads, with $v_{80 \%} \approx 0.18$. 

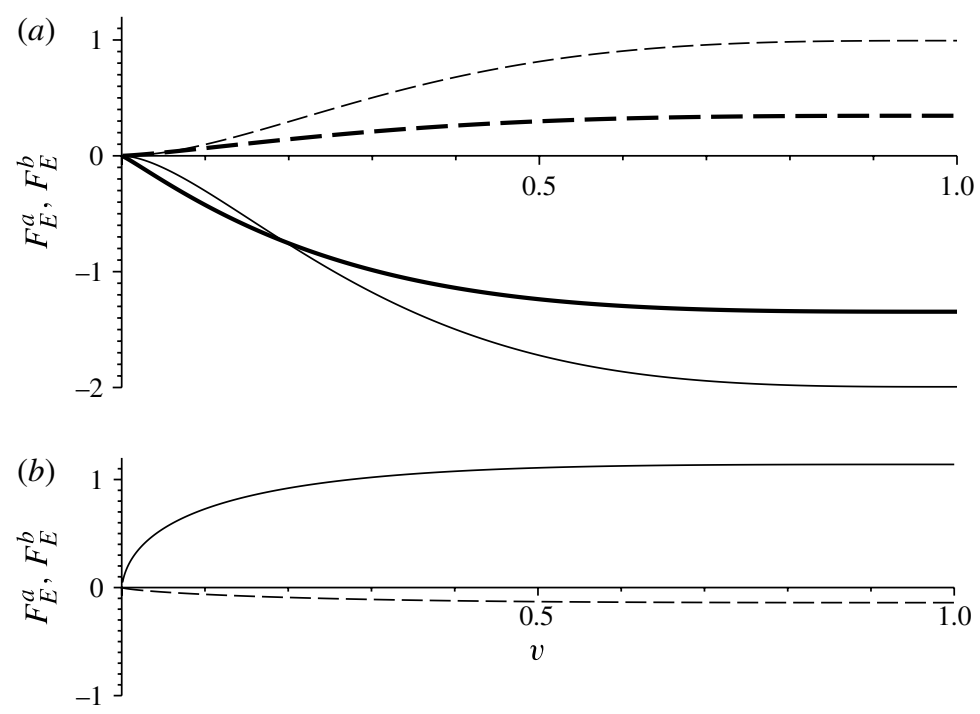

FIGURE 5. Contributions to the flux fraction from type a triads (dashed lines) and type $\mathrm{b}$ triads (solid lines) for $(a) \alpha=1$ (thin lines) and $\alpha=2$ (thick lines) and (b) $\alpha=3$.

Figure 5 shows the total flux fraction decomposed into contributions from type a and type $\mathrm{b}$ triads. For $\alpha=1$ and $\alpha=2$, type a triads contribute downscale flux and type $\mathrm{b}$ triads upscale flux. This is in agreement with, for example, the numerical results of Smith \& Yakhot (1994), who found that one term in Kraichnan's decomposition contributed entirely negative and the other entirely positive flux for $\alpha=2$. For $\alpha=3$, the situation is opposite, with type a triads contributing upscale flux and type $b$ triads downscale flux. Since type $b$ triads dominate, the net flux is downscale in this case.

In figure 6 , the variation of $v_{80 \%}$ with $\alpha$ is shown for the total energy flux, as well as the contributions from type a and type b triads. Cancellations between these two contributions to the flux result in the total flux being less local than either the type a or type $\mathrm{b}$ fluxes taken alone. Type a triads are more local than type $\mathrm{b}$ triads, as must be the case: these triads involve wavevectors $\boldsymbol{k}^{\prime}, \boldsymbol{p}, \boldsymbol{q}$, with $p, q<k<k^{\prime}$, where the flux is past $k$. Since $p, q<k$, the requirement that the triad close places a restriction on how large $k^{\prime}$ can be. As $\alpha \rightarrow 4, v_{80 \%}$ drops precipitously toward zero, in accordance with the divergence of the energy flux at $\alpha=4$. (Again, $\alpha>3$ may not be physically relevant.)

Figure 7 shows the energy flux, modulo $\varepsilon C^{3 / 2} \mu^{-1}$, associated with the spectrum (2.3a) as a function of $\alpha$ for the total flux, as well as the flux contributions from type $\mathrm{a}$ and type $\mathrm{b}$ triads. All three fluxes vanish at $\alpha=5 / 2=2.5$ and change sign thereafter. Moreover, the EDQNM triad transfer function vanishes identically at $\alpha=2.5$, so not only is there no net flux associated with the self-similar inertial range for this value of $\alpha$, there are no turbulent transfers at all.

Hence, for $\alpha>2.5$, the EDQNM closure, combined with the similarity assumption that allowed us to rescale the triad transfer function, gives surprising results. At $\alpha=2.5$, the energy flux vanishes identically, and for $\alpha>2.5$, the net energy flux is downscale. Specifically, the quantity that vanishes and then changes sign is $\left[2 a_{1 v w}(v w)^{-(7-\alpha) / 3}-b_{1 v w} v w^{-(7-\alpha) / 3}-b_{1 w v} w v^{-(7-\alpha) / 3}\right]$ in (5.29). The weight 


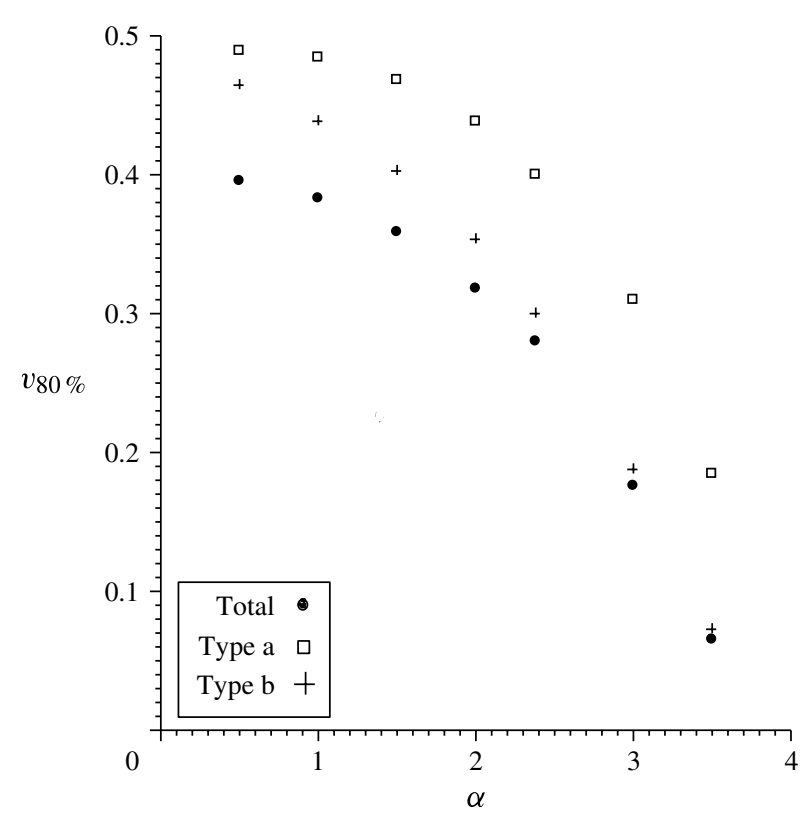

FIGURE 6. The variation of $v_{80 \%}$ as a function of $\alpha$.

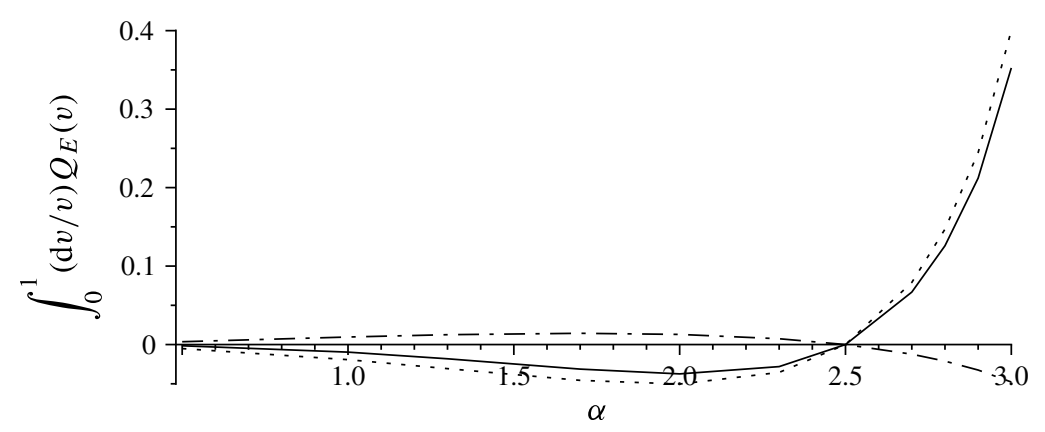

FIGURE 7. Sign of the energy flux as a function of $\alpha$. The dash-dot line is the contribution from type a triads, the dotted line the contribution from type $b$ triads, and the solid line the total.

function $W_{E}[v, w,(7-\alpha) / 3]$ remains positive, and its two terms retain their respective signs. This explains why both $\pi_{E}^{a}$ and $\pi_{E}^{b}$ change sign at the same value of $\alpha$.

Taking into account the fact that very non-local triads exchange most of their energy with smaller scales (see table 1), combined with the fact that the EDQNM generalized energy flux is increasingly dominated by non-local transfers as $\alpha$ increases, it is tempting to attribute the positive (downscale) flux for $\alpha>2.5$ to dominantly non-local transfers. However, the net energy and enstrophy transfer by a given triad, calculated from Fjørtoft arguments, depends only on triad geometry and the triad conservation laws. The net contribution to the flux in the EDQNM approximation depends on triad geometry, the triad conservation laws, a similarity assumption and a closure. Hence, 
it is difficult to draw a straightforward comparison between the results of the Fjørtoft arguments and the predictions of the EDQNM closure.

In appendix E, we show that energy transfer is out of the middle wavenumber in the self-similar energy-cascading inertial range, with $(5.16 a)$ and $(5.16 b)$, for $\alpha \notin(2.5,10)$, while it is into the middle wavenumber for $\alpha \in(2.5,10)$. This may account for the change in sign of the energy flux.

\section{Statistical mechanical equilibrium spectra and cascade directions}

It has been argued that turbulent inertial range fluxes represent the system's attempt to reach a state of statistical mechanical equilibrium, as defined by maximization of some appropriate entropy (e.g. Kraichnan 1967, 1975). Kraichnan (1967) derived statistical equilibrium spectra for inviscid, unforced, spectrally truncated Navier-Stokes $(\alpha=2)$ flow. He argued for downscale enstrophy flux and upscale energy flux by comparing the equilibrium enstrophy spectrum with the spectrum $(2.3 a)$ in the enstrophy inertial range. The statistical equilibrium spectra correspond to Gaussian zero-mean ensembles, which are exact statistical solutions for spectrally truncated 2D fluids at zero viscosity because the Fourier amplitudes satisfy a Liouville theorem and thus conserve probability (Orszag 1970; Fox \& Orszag 1973; Kraichnan 1975).

In an attempt to gain insight into the directions of the energy and enstrophy fluxes associated with our self-similar inertial range solutions, we thus compare their spectra with absolute equilibrium distributions for inviscid, spectrally truncated models. As before, we work in a doubly periodic box of side length $L$. The total energy and enstrophy are

$$
\begin{aligned}
& \hat{E}=\frac{1}{2} \overline{\psi \theta}=\sum_{\boldsymbol{k}} k^{-\alpha}|\hat{\theta}(\boldsymbol{k})|^{2}, \\
& \hat{Z}=\frac{1}{2} \overline{\theta^{2}}=\sum_{\boldsymbol{k}}|\hat{\theta}(\boldsymbol{k})|^{2},
\end{aligned}
$$

where the overline denotes a volume average. We define

$$
k_{*}^{\alpha}=\frac{\hat{Z}}{\hat{E}} .
$$

The grand canonical ensemble

$$
P \propto \exp \left[-\sum_{k}\left(a k^{-\alpha}+b\right)|\hat{\theta}(\boldsymbol{k})|^{2}\right]
$$

maximizes the entropy subject to the constraint that

$$
2(a \hat{E}+b \hat{Z}) \equiv \sum_{k}\left(a k^{-\alpha}+b\right)|\hat{\theta}(\boldsymbol{k})|^{2}
$$

is conserved, yielding

$$
\left\langle|\hat{\theta}(\boldsymbol{k})|^{2}\right\rangle=\frac{1}{2} \frac{k^{\alpha}}{a+b k^{\alpha}} .
$$

If the system is truncated such that $k_{\min }<k<k_{\max }$, and $k_{\min }$ is large enough that the modes are dense over the entire spectrum, then we may use the isotropic expressions 
for the energy spectrum (5.5) and the corresponding expression for the isotropic enstrophy spectrum. This gives statistical equilibrium distributions

$$
\begin{aligned}
& \mathscr{E}_{e q}(k)=\frac{\pi}{2} \frac{k}{a+b k^{\alpha}}, \\
& \mathscr{Z}_{e q}(k)=\frac{\pi}{2} \frac{k^{\alpha+1}}{a+b k^{\alpha}} .
\end{aligned}
$$

For any realizable values of $\hat{E}$ and $\hat{Z}$ such that $k_{\min }^{\alpha}<k_{*}^{\alpha}<k_{\max }^{\alpha}$, it is possible to find unique $a$ and $b$ such that (7.6a) and (7.6b) give the required $\hat{E}$ and $\hat{Z}$, and $\mathscr{E}_{e q}(k)$, $\mathscr{Z}_{e q}(k)$ are positive. This was shown for $\alpha=2$ by Salmon, Holloway \& Hendershott (1976), whose proof is easily generalized to $\alpha>0$, and by Fox \& Orszag (1973). For $a=0$, enstrophy is in equipartition, while for $b=0$, energy is in equipartition.

We first compare the equipartition enstrophy spectra with the KLB spectra (2.3a) in the enstrophy inertial range to see if the relationship between these spectra is associated with a particular flux direction. Note that we do not expect to observe the KLB spectra for $\alpha>2$ : the generalized enstrophy inertial range will instead exhibit a passive tracer spectrum. Our purpose at the moment is to study the flux sign associated with the KLB range solution, whether or not this solution is physically relevant.

Note that the statistical equilibrium spectra $(7.6 a)$ and (7.6b) are equilibria of the EDQNM closure (Orszag 1977; Kraichnan \& Montgomery 1980). This can be confirmed by substituting (7.6a) and (7.6b) into (5.4): $T_{E}(k, p, q)$ vanishes identically in each case. Note also that we made no similarity assumptions in deriving (5.4). In contrast, to obtain (5.29) and (5.33) we made similarity assumptions that require the energy spectrum to have a power-law form $(5.16 a)$. The only statistical equilibrium solutions with power-law form are the equipartition solutions, for which $a=0$ or $b=0$ in $(7.6 a)$ and $(7.6 b)$. Hence, only the equipartition solutions can be equilibrium solutions of (5.29) and (5.33).

Also, we note that the EDQNM solutions obey an $\mathrm{H}$-theorem, which drives the inviscid unforced evolution toward the equilibrium solutions (7.6a) and (7.6b) while entropy increases monotonically (Carnevale, Frisch \& Salmon 1981). In general, there is no $\mathrm{H}$-theorem driving inviscid unforced evolution toward the similarity solutions. As such, the equilibrium solutions have a special status and stability not shared by the similarity solutions.

The left-hand side of table 2 shows the KLB spectra and associated flux signs in the enstrophy inertial range, with positive flux indicating net transfer toward smaller scales, as before. For $\alpha=2$ and $\alpha=3$, the KLB spectrum is steeper than the equipartition spectrum, $\mathscr{Z}_{e q} \propto k$, which means the large- $k$ modes have less enstrophy than they would in equipartition. Downscale flux for these values of $\alpha$ is consistent with the idea that the system is attempting to reach enstrophy equipartition by increasing the enstrophy at smaller scales. For $\alpha=6$, in contrast, the KLB spectrum is shallower than the equipartition spectrum, and the flux is upscale, consistent with an attempt to reach equipartition by removing excess enstrophy at smaller scales. Furthermore, when the equipartition and KLB spectra coincide at $\alpha=5$, the flux vanishes, as does $T_{E}(k, p, q)$. This means that, for $\alpha=5$, the KLB spectrum corresponds to equipartition, and a system exhibiting the KLB spectrum would be in equilibrium, with vanishing turbulent transfers and Gaussian statistics.

The right-hand side of table 2 shows the KLB spectra in the energy inertial range. In contrast to the enstrophy inertial range, there are two values of $\alpha$ at which the energy flux and triple correlation function vanish identically. These are $\alpha=2.5$ and 


$\begin{array}{lccclccc}\alpha & \mathscr{E}(k) & \mathscr{Z}(k) & \operatorname{sgn}\left(\pi_{Z}\right) & \alpha & \mathscr{E}(k) & \mathscr{Z}(k) & \operatorname{sgn}\left(\boldsymbol{\pi}_{E}\right) \\ 2 & k^{-3} & k^{-1} & + & 2 & k^{-5 / 3} & k^{1 / 3} & - \\ 2.5 & k^{-19 / 6} & k^{-2 / 3} & + & \mathbf{2 . 5} & \boldsymbol{k}^{-\mathbf{1 . 5}} & \boldsymbol{k} & \mathbf{0} \\ 3 & k^{-10 / 3} & k^{-1 / 3} & + & 3 & k^{-4 / 3} & k^{5 / 3} & + \\ \mathbf{5} & \boldsymbol{k}^{-\mathbf{4}} & \boldsymbol{k} & \mathbf{0} & 5 & k^{-2 / 3} & k^{13 / 3} & + \\ 6 & k^{-13 / 3} & k^{5 / 3} & - & 6 & k^{-1 / 3} & k^{18 / 3} & + \\ 9 & k^{-16 / 3} & k^{11 / 3} & - & 9 & k^{2 / 3} & k^{29 / 3} & + \\ 10 & k^{-17 / 3} & k^{13 / 3} & - & \mathbf{1 0} & \boldsymbol{k} & \boldsymbol{k}^{11} & \mathbf{0} \\ 11 & k^{-6} & k^{5} & - & 11 & k^{4 / 3} & k^{37 / 3} & -\end{array}$

TABLE 2. Wavenumber dependence of KLB spectra in the enstrophy (left) and energy (right) inertial ranges, with associated flux signs as calculated using the EDQNM closure. A zero indicates vanishing flux. The bold rows correspond to equipartition of energy or enstrophy.

$\alpha=10$. At these values of $\alpha$, the KLB spectra correspond to enstrophy and energy equipartition, respectively. The sign of the flux in the energy inertial range thus depends on the relationships between the KLB spectra and both the energy and enstrophy equipartition spectra. The energy flux first changes sign from upscale to downscale at $\alpha=2.5$, where the KLB spectrum corresponds to enstrophy equipartition. For $\alpha<2.5$ the KLB spectrum is steeper than the spectrum associated with enstrophy equipartition, and the energy flux is upscale. For $\alpha>2.5$, the spectrum is shallower than that associated with enstrophy equipartition, but steeper than that associated with energy equipartition, and the energy flux is downscale. When, at $\alpha=10$, the KLB spectrum shallows past that associated with energy equipartition, the energy flux changes sign again, from downscale to upscale. Again, $\alpha>3$ may be unphysical, but results up to $\alpha=11$ are included in order to show the second absolute equilibrium at $\alpha=10$, and the change in sign of the energy flux thereafter.

Figure 8 shows a plot of $Q_{E}(v, w)$ and $Q_{Z}(v, w)$ for $v=0.95$ and $w=1.85$. Both quantities are scaled by $\alpha^{-2}$ so that all zeros are clearly visible. Here $Q_{E}(v, w)$ and $Q_{Z}(v, w)$, defined in (5.29) and (5.33), have the same signs for all non-zero $v, w$, and the same signs as their respective triad transfer functions and fluxes. Hence, figure 8 effectively shows the signs of the energy and enstrophy fluxes associated with the similarity solutions for various $\alpha$.

In summary, the flux directions associated with the similarity solutions reflect the relationship between the KLB spectra and the equipartition spectra. The zero flux solutions are self-similar inertial ranges in which $(5.16 a)$ and $(5.16 b)$ hold, the triad transfer function vanishes, the statistics are Gaussian, and the KLB and equipartition spectra coincide. Note again that, since we have imposed the requirement that the energy spectrum be a power law, $\mathscr{E}(k) \propto k^{-n}$, the equipartition spectra are the only equilibrium solutions we will find.

\section{Steepening past the KLB spectrum in the inverse cascade for $\alpha \geqslant 2.5$}

The locally dominated strain rate for $\alpha<4$, eddy turnover time decreasing with $k$ for $\alpha<4$, and the fact that the EDQNM energy flux associated with the self-similar (as defined by (5.16a) and (5.16b)) inertial range does not diverge until $\alpha=4$ all suggest that the energy cascade should exhibit the KLB spectrum for $\alpha<4$. However, the association of the self-similar inertial range solution with vanishing energy flux 


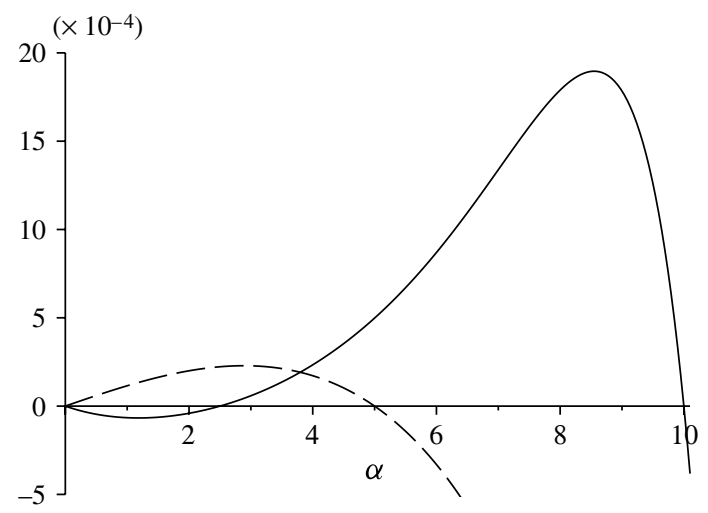

FIGURE 8. Sign of the triad transfer function and flux as a function of $\alpha$ for similarity solutions calculated using the EDQNM closure. The dashed line is $Q_{Z}(0.95,1.85) / \alpha^{2}$ and the solid line is $Q_{E}(0.95,1.85) / \alpha^{2}$.

for $\alpha=2.5$ and downscale energy flux for $2.5<\alpha<10$ suggests that, if an inverse cascade is observed for $\alpha \geqslant 2.5$, it will deviate from KLB phenomenology and not show the spectrum $(2.3 a)$.

To test this, we ran a series of turbulence simulations with code at resolution $512^{2}$ (maximum wavenumber $k=256$ ) in a doubly periodic domain for values of $\alpha$ between 2 and 3 . The code is pseudospectral, with full two-thirds spectral dealiasing, so $k_{\max }=N / 3$, where $N$ is the grid resolution in $x$ and $y$. The initial condition is a state of no flow. The forcing is $\delta$ correlated in time and band-limited in spectral space, with non-zero forcing $f$ in the band $\left|k-k_{f}\right| \leqslant \Delta k=k_{f} / 16$, where $k_{f}$ is the forcing wavenumber and $f=0$ otherwise. Time stepping is by a fourth-order Runge-Kutta scheme. We use small-scale diffusion but no damping at large scales, so the turbulence is in a quasistationary state, in which the integral scale grows with time. Data were taken from times before the energetic peak hit the domain scale.

A developing literature (Scott 2007; Vallgren 2011) indicates that the inverse cascade spectrum for $\alpha=2$ steepens beyond the KLB prediction when the forcing scale is well-resolved, probably due to coherent vortex formation at inverse cascade scales. Forcing in the dissipation range, in contrast, suppresses enstrophy generation and produces the KLB spectrum. Thus, to eliminate spectral steepening due to coherent vortex formation and test whether it is possible to generate the KLB spectrum, the systems were forced at $k_{f}=170$. We first found a value of the small-scale diffusion for which energy was largely dissipated at the forcing scale, the vorticity field remained dominated by forcing noise until the end of the run, and no nonlinear interactions or inverse cascade developed. The diffusion was then decreased until a well-developed inverse cascade, as defined by a broad spectral range with an approximately $k$-independent energy flux, appeared by the end of the run, and nonlinear advection visibly dominated the vorticity field.

Figures 9 and 10 show spectra averaged over 10 consecutive time steps for these runs. As evident in figure 9, inverse cascades with spectra well-fit by the KLB prediction develop for $\alpha=2, \alpha=2.2$ and $\alpha=2.4$, consistent with the EDQNM results. In contrast, even forcing at $k_{f}=170$ and using the largest diffusion such that an inverse cascade develops does not produce the KLB spectrum for $\alpha=2.5$ and larger (figure 10). Instead, the energy spectral slope is visibly steeper, and steepens 


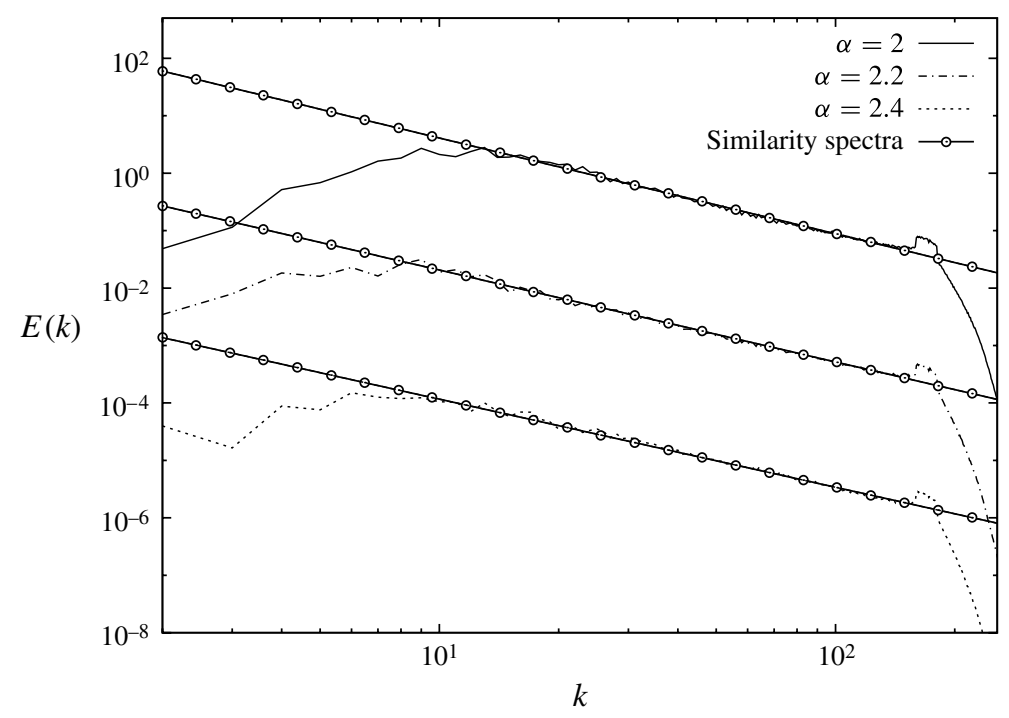

FIGURE 9. Energy spectra averaged over 10 consecutive time steps in the inverse cascade for $\alpha=2$ (solid), $\alpha=2.2$ (dash-dot) and $\alpha=2.4$ (dotted) with KLB spectra fits.

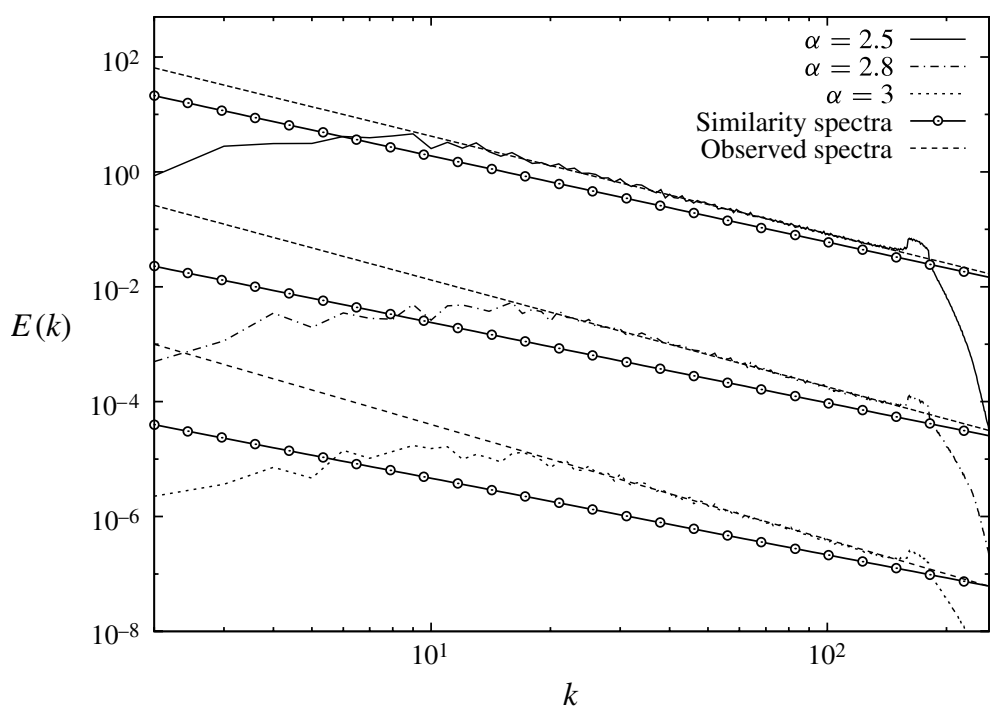

FIGURE 10. Energy spectra averaged over 10 consecutive time steps in the inverse cascade for $\alpha=2.5$ (solid), $\alpha=2.8$ (dash-dot) and $\alpha=3$ (dotted) with linear fits and KLB spectra for contrast. The slopes of the linear fits are $-1.7(\alpha=2.5),-1.86(\alpha=2.8)$ and $-2.0(\alpha=3)$.

as $\alpha$ increases, with spectral slopes of -1.7 for $\alpha=2.5,-1.86$ for $\alpha=2.8$ and -2.0 for $\alpha=3$. Hence, the EDQNM prediction that the inverse energy cascade will not satisfy KLB phenomenology for $\alpha \geqslant 2.5$ is numerically supported, even in these 


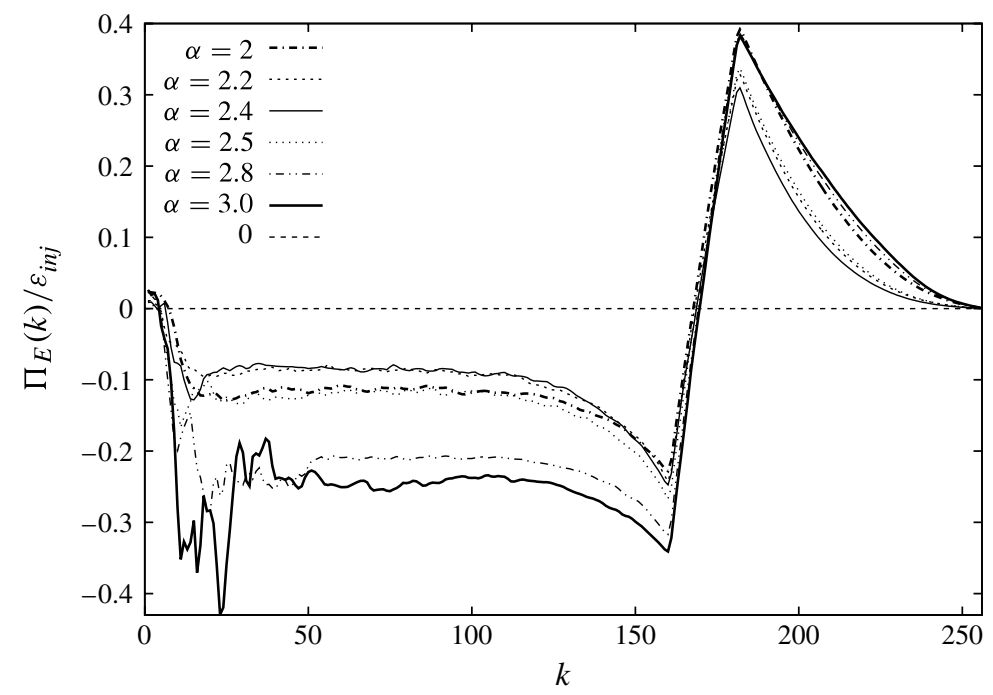

FIGURE 11. Energy fluxes, scaled by the rate of energy injection $\varepsilon_{i n j}$, corresponding to the spectra in figures 9 and 10 .

low-resolution runs. Furthermore, and in contrast, we do obtain the KLB spectrum for $\alpha<2.5$ with sufficiently large $k_{f}$.

Note that visual inspection of the vorticity fields that develop with our choices of forcing and diffusion reveals no coherent vortices, so these are not responsible for the spectral steepening. Further, the spectral slopes remain the same for a range of diffusions and large forcing wavenumbers. In fact, for $\alpha=3$ a test run at $k_{f}=64$ produced an inverse cascade with the same spectral slope, -2.0 , and very few, small and short-lived vortices. This contrasts with $\alpha=2$ forced at $k_{f}=64$, for which a population of long-lived coherent vortices develops and the spectrum steepens past the KLB prediction obtained at $k_{f}=170$. Numerical work on the physical and spectral space characteristics of the inverse cascade for various $\alpha$ is in progress.

Using (2.6) and the measured slopes, one finds that the predictability time associated with the simulated power laws is finite for $\alpha=2.5$ and $\alpha=2.8$ and diverges logarithmically for $\alpha=3$. In contrast, as shown in $\S 2$, for the KLB spectrum $(2.3 a)$, the predictability time in the inverse cascade does not diverge until $\alpha=4$, for which the divergence is logarithmic.

Figure 11 shows the energy fluxes, also averaged over 10 consecutive time steps, corresponding to these spectra. The fluxes are scaled by the energy injection rate, which varies with $\alpha$, for better comparison. The flux is upscale in the inertial range for all values of $\alpha$. Inverse-cascading inertial ranges with nearly constant fluxes are visible. The large variations in the flux at small $k$ are due to a lack of statistics. There is some indication that more of the injected energy cascades upscale as $\alpha$ increases, but caution must be used in comparing the fluxes, since their magnitude, unlike the spectral slopes, is quite sensitive to $k_{f}$ and the choice of diffusion. Further and more careful study is required to adequately characterize the strengths of the inverse cascades for various $\alpha$. The large downscale fluxes are due to forcing in the dissipation range, and are much reduced when the systems are forced at larger scales. 


\section{Discussion and conclusions}

In this paper, we examined the extent to which classical turbulence phenomenology describes energy cascades in 2D fluids. The $\alpha$ turbulence models we studied are characterized by varying degrees of spectrally non-local advection, and include several simplified geophysical systems. Our focus was on exploring parameter space to assess various common arguments about $2 \mathrm{D}$ turbulence, including those for dual cascades and for the validity of self-similar KLB inertial range phenomenology.

We reviewed some arguments traditionally used to support the expectation of dual inverse energy and direct enstrophy cascades in two dimensions. These include Fjørtoft arguments, as well as a similarity argument and an energy-enstrophy conservation argument, both of which rely on having an initially spectrally localized disturbance spreading in spectral space. The energy-enstrophy conservation argument can be extended to $\alpha \neq 2$, showing that, under such initial conditions, energy must be transferred to larger scales and enstrophy to smaller scales for $\alpha>0$. Although they have been used to motivate expectations of dual cascades, these arguments do not extend to statistically steady infinite Reynolds number inertial ranges, in which energy and enstrophy are not spectrally localized. They thus cannot be used to predict energy and enstrophy flux directions in the infinite self-similar inertial ranges considered in classical turbulence phenomenology. These arguments do, however, apply to numerical simulations in which steady or quasisteady inertial ranges develop due to energy and enstrophy injection by a spectrally localized forcing.

Following Merilees \& Warn (1975), we generalized Fjørtoft arguments to positive values of $\alpha$. The directions of net energy and enstrophy transfer due to a particular triad interaction depend on triad geometry and whether transfer is into or out of the middle wavenumber. Moderately local triads exchange more energy with large scales and more enstrophy with small scales for all values of $\alpha$, and the percentages increase with $\alpha$. On the other hand, non-local triads exchange both more energy and more enstrophy with small scales. For transfer out of the middle wavenumber, which is a reasonable assumption given an initially localized disturbance spreading in spectral space, this means that moderately local triads contribute net upscale energy and downscale enstrophy transfer, while non-local triads contribute both net downscale energy and net downscale enstrophy transfer. Indeed, as explicitly demonstrated, Fjørtoft arguments allow transfers counter to common intuition. As shown, it is possible to construct two triads engaging in transfers that respect the triad conservation laws, and which result in net downscale energy transfer and zero enstrophy transfer. As noted also by previous authors (Merilees \& Warn 1975; Tung \& Welch 2001; Gkioulekas \& Tung 2007), Fjørtoft arguments alone cannot be used to predict energy and enstrophy cascade directions. First, such geometrical arguments yield no information on triad dynamical activity, i.e. how much various triads contribute to the fluxes. Second, it is not clear, in a statistically steady inertial range, whether transfer will be into or out of the middle wavenumber on average; in general, transfers of both senses will occur.

An EDQNM closure allows us to add dynamics in first approximation, and to investigate the sense of energy and enstrophy transfers in triad interactions. We make similarity assumptions for the energy spectrum and triad transfer function. In so doing, we seek self-similar inertial range solutions, recovering the phenomenological predictions (2.3a) and (2.3b) upon requiring $k$-independent flux. We then study the characteristics of these self-similar inertial ranges, including degree of spectral nonlocality and flux directions, with a view to exploring whether 2D energy cascades can be expected to exhibit the solutions predicted by classical KLB phenomenology. 
We emphasize again that coherent structures and any effects they may have on inertial range phenomenology are beyond the scope of the present study. The EDQNM solutions are approximate solutions that filter out coherent structures. Depending on how important these structures are for a given $\alpha$ and choice of simulation parameters, the EDQNM solutions may be a better or worse representation of the flow. As mentioned previously, for $\alpha=2$, it is known that the turbulent background flow obtained upon filtering out coherent structures displays the KLB spectrum. The numerical simulations presented in $\S 8$ show that we obtain the spectrum $(2.3 a)$ for small-scale forcing and $2 \leqslant \alpha<2.5$, but not for $\alpha \geqslant 2.5$. It is the characteristics of this background flow, including whether or not it can exist and exhibit the KLB scaling for various $\alpha$, that we hope to gain insight into using the EDQNM.

In the KLB energy cascade predicted by phenomenological arguments, the strain rate is locally dominated and the eddy turnover time decreases with $k$ for $k<4$, becoming $k$-independent at $\alpha=4$. In the enstrophy cascade, the eddy turnover time becomes $k$-independent at $\alpha=2$. In keeping with the behaviour of the eddy turnover time, the energy flux calculated from the EDQNM closure and similarity assumption is finite for $\alpha<4$ and dominated by contributions from $v>0$, where $1, v, w$ is a triad shape, with $0<v<1$ and $1<w<1+v$. The contributions to the energy flux from non-local triads become more important as $\alpha$ increases. The energy flux becomes unavoidably non-local and diverges at $\alpha=4$, dominated by contributions from $v \rightarrow 0$. In keeping with previous results, and with the behaviour of the eddy turnover time, the EDQNM enstrophy flux becomes unavoidably non-local and dominated by infrared contributions at $\alpha=2$. When the fluxes are dominated by contributions from $v \rightarrow 0$, the infrared cutoff will determine the dynamics, no matter the system size, and the cascade is non-local.

The locally dominated strain rate, eddy turnover time decreasing with $k$, and finite energy flux dominated by triads with $v>0$ seem to indicate that the energy-cascading inertial range should exhibit KLB self-similar inertial range phenomenology for $\alpha<4$. However, for $\alpha=2.5$, the triad energy transfer function and energy flux associated with the similarity range vanish identically, and for $\alpha>2.5$, the self-similar inertial range solution, as defined by $(5.16 a)$ and $(5.16 b)$, has downscale energy flux. One can prove (see appendix $\mathrm{E}$ ) that transfer is out of the middle wavenumber in the self-similar energy inertial range for $\alpha \notin(2.5,10)$, and into the middle wavenumber for $\alpha \in(2.5,10)$, which seems to account for the changes in flux sign.

A direct-cascading energy inertial range would require zero enstrophy transfer through the inertial range and enstrophy dissipation at a rate $k_{d}^{\alpha} \varepsilon$, i.e. enstrophy would have to be dissipated at a far greater rate than it was being transferred. Hence, such a direct-cascading energy range is not physically realizable. This implies that any inverse energy cascades observed for $\alpha \geqslant 2.5$ will not exhibit KLB phenomenology. Numerical simulations confirm this prediction, showing that the energy spectrum in the inverse cascade for $\alpha \geqslant 2.5$ is distinctly steeper than the KLB spectrum. In contrast, for $\alpha<2.5$ the KLB power law fits the inverse cascade energy spectrum well, consistent with the EDQNM prediction.

As $\alpha$ decreases to 1 , the tendency to form coherent structures increases, even with forcing near the dissipation range. However, upon filtering out the vortex cores, we find in preliminary numerical simulations that the background retains the KLB spectrum. Numerical work on the formation of coherent structures in the inverse energy cascade for $0<\alpha \leqslant 3$ is in progress.

From (2.6), we showed that for the KLB spectrum $(2.3 a)$, the predictability time in the inverse energy cascade diverges logarithmically when $\alpha=4$. This would lead us to 
expect finite predictability time for $\operatorname{RSF}(\alpha=3)$ if the similarity theory held for that model. However, from our numerical simulations, we find that the spectral slope in the RSF inverse cascade is -2.0 , which yields a logarithmically divergent predictability time. Hence, the RSF inverse cascade is more predictable than the similarity theory would indicate, which has practical implications for model truncation.

Eddy turnover time and strain rate alone obviously fail to predict the physical realizability of KLB energy cascades in two dimensions. This may signal a poor understanding of the mechanisms by which inverse energy transfer occurs in $2 \mathrm{D}$ flows. The direction of the flux associated with self-similar inertial range solutions satisfying (5.16a) and (5.16b) reflects the relationship between equipartition spectra calculated for spectrally truncated inviscid fluids and the KLB spectra. The triad transfer functions and fluxes vanish when the KLB spectra coincide with equipartition spectra. For the energy inertial range, this occurs at $\alpha=2.5$, where the KLB spectrum corresponds to enstrophy equipartition, and at $\alpha=10$, where the similarity solution corresponds to energy equipartition. As $\alpha$ increases past 2.5, the energy flux and triad energy transfer function change sign from negative (upscale) to positive (downscale), and as $\alpha$ increases past 10, the triad transfer function changes sign from positive to negative again. (Recall that, for $\alpha \geqslant 4$ and the KLB spectrum, the energy flux diverges in the limit of an infinite inertial range, so the triad transfer function is the appropriate quantity to examine for $\alpha>4$.) Our results underline the important relationship between statistical equilibrium solutions and turbulent cascade phenomenology and realizability in two dimensions.

\section{Acknowledgements}

We would like to thank three anonymous reviewers for their constructive suggestions, and particularly reviewer 3 for providing the derivation of (5.14) upon which appendix D is based. R. K. Scott kindly shared his turbulence code, which we used for the simulations. We thank $T$. Iwayama for a careful reading of the manuscript, useful comments, and for pointing out a simpler way to determine the sign of $T_{E}(k, p, q)$, which is presented in appendix E. Funding was provided by the Natural Sciences and Engineering Research Council of Canada. B.H.B. was supported by an NSERC Canada Graduate Scholarship and an Amelia Earhart Fellowship from the Zonta International Foundation.

\section{Appendix A}

Consider

$$
\frac{\partial \tilde{\theta}}{\partial \tilde{t}}+\tilde{J}(\tilde{\psi}, \tilde{\theta})=0
$$

where $\tilde{\theta}=(-\tilde{\Delta})^{\alpha / 2} \tilde{\psi}, \alpha$ can be of either sign, and the tildes indicate that all variables are now non-dimensionalized.

Introduce the scaling transformation

$$
\tilde{t} \rightarrow \beta^{r} \tilde{t}, \quad \tilde{x} \rightarrow \beta^{s} \tilde{x}, \quad \tilde{\psi} \rightarrow \beta^{p} \tilde{\psi}, \quad \tilde{\theta} \rightarrow \beta^{q} \tilde{\theta},
$$

where $\beta$ is non-dimensional. The governing equation becomes

$$
\beta^{q-r} \frac{\partial \theta}{\partial t}+\beta^{p+q-2 s} J(\psi, \theta)=0,
$$


where we have dropped the tildes. For the dynamics to be self-similar, the exponents of $\beta$ must match. Hence,

$$
p=2 s-r .
$$

Similarly,

$$
\beta^{q} \theta=\beta^{-\alpha s} \beta^{p}(-\Delta)^{\alpha / 2} \psi,
$$

and we require

$$
q=p-\alpha s .
$$

For an energy inertial range, let the energy spectrum have the form

$$
\mathscr{E}(k)=C \varepsilon^{n} k^{m},
$$

where $\varepsilon$ is a non-dimensionalized energy flux. We seek values of $n$ and $m$ corresponding to scaling solutions. Under (A 2),

$$
E \rightarrow \beta^{p+q} E=\beta^{(4-\alpha) s-2 r} E,
$$

and

$$
\mathscr{E}(k) \rightarrow \beta^{p+q+s} \mathscr{E}(k)=\beta^{(5-\alpha) s-2 r} \mathscr{E}(k) .
$$

The flux becomes

$$
\varepsilon \rightarrow \beta^{p+q-r} \varepsilon=\beta^{(4-\alpha) s-3 r} \varepsilon .
$$

Using (A 9), (A 10) and $k \rightarrow \beta^{-s} k$, we obtain

$$
\beta^{(5-\alpha) s-2 r} \mathscr{E}(k)=C \beta^{[(4-\alpha) s-3 r] n-s m} \varepsilon^{n} k^{m} .
$$

We now seek solutions where space and time scale independently, so that $r$ and $s$ are independent. Then the coefficient of $r$ on the left-hand side of (A 11) must match the coefficient of $r$ on the right-hand side, and similarly for $s$. This yields

$$
\begin{array}{rlrl}
-2=-3 n & \Rightarrow n=2 / 3 & & \text { (coefficient of } r), \\
5-\alpha=2(4-\alpha) / 3-m & \Rightarrow m=-(7-\alpha) / 3 & \text { (coefficient of } s) .
\end{array}
$$

Hence, the similarity solution for the energy spectrum in the energy inertial range is

$$
\mathscr{E}(k)=C \varepsilon^{2 / 3} k^{-(7-\alpha) / 3},
$$

in agreement with $(2.3 a)$.

In the enstrophy inertial range, let

$$
\mathscr{E}(k)=C^{\prime} \eta^{n^{\prime}} k^{m^{\prime}} .
$$

Under (A 2),

$$
Z \rightarrow \beta^{2 q} Z=\beta^{2(2-\alpha) s-2 r} Z,
$$

and

$$
\eta \rightarrow \beta^{2 q-r} \eta=\beta^{2(2-\alpha) s-3 r} \eta .
$$

Using (A 9) and (A 16), we obtain

$$
\beta^{(5-\alpha) s-2 r} \mathscr{E}(k)=C^{\prime} \beta^{[2(2-\alpha) s-3 r] n^{\prime}-s m^{\prime}} \eta^{n^{\prime}} k^{m^{\prime}} .
$$


Requiring space and time to scale independently,

$$
\begin{array}{cc}
-2=-3 n^{\prime} \Rightarrow n^{\prime}=2 / 3 & \text { (coefficient of } r), \\
5-\alpha=2(4-2 \alpha) / 3-m^{\prime} \Rightarrow m^{\prime}=-(7+\alpha) / 3 & \text { (coefficient of } s) .
\end{array}
$$

This yields the KLB spectrum

$$
\mathscr{E}(k)=C^{\prime} \eta^{2 / 3} k^{-(7+\alpha) / 3}
$$

for the energy spectrum in the enstrophy-cascading inertial range, in agreement with (2.3b). For $\alpha=-2$, (A 13) yields $\mathscr{E}(k) \propto k^{-3}$ and $\mathscr{Z} \propto k^{-5}$, while (A 19) yields $\mathscr{E}(k) \propto k^{-5 / 3}$ and $\mathscr{Z}(k) \propto k^{-11 / 3}$. This agrees with previous results (Larichev \& McWilliams 1991; Boffetta, De Lillo \& Musacchio 2002), where we note that generalizing the formalism to both positive and negative $\alpha$ has led us to exchange the usual definitions of energy and enstrophy taken in Charney-Hasegawa-Mima turbulence.

\section{Appendix B}

For general $\alpha$, we obtain

$$
\begin{aligned}
S_{E} & \equiv \frac{X_{E}}{Y_{E}}=\frac{q^{\alpha}-p^{\alpha}}{p^{\alpha}-k^{\alpha}}=\frac{|\boldsymbol{k}-\boldsymbol{p}|^{\alpha}-p^{\alpha}}{p^{\alpha}-k^{\alpha}} \\
& =\frac{(\boldsymbol{k} \cdot \boldsymbol{k}-2 \boldsymbol{k} \cdot \boldsymbol{p}+\boldsymbol{p} \cdot \boldsymbol{p})^{\alpha / 2}-p^{\alpha}}{p^{\alpha}-k^{\alpha}} \\
& =\frac{\left(k^{2}+2 k p \cos \phi+p^{2}\right)^{\alpha / 2}-p^{\alpha}}{p^{\alpha}-k^{\alpha}}
\end{aligned}
$$

where $\phi$ is the angle between $\boldsymbol{k}$ and $-\boldsymbol{p}$. Since $q \geqslant p$ and $p \geqslant k, S_{E} \geqslant 0$. If we divide the numerator and denominator of $S_{E}$ by $p^{\alpha}$, we get

$$
S_{E}=\frac{\left(v^{2}+2 v \cos \phi+1\right)^{\alpha / 2}-1}{1-v^{\alpha}},
$$

since $v=k / p$, where

$$
S_{E} \geqslant 0 \Rightarrow\left(v^{2}+2 v \cos \phi+1\right)^{\alpha / 2} \geqslant 1 \Rightarrow \cos \phi \geqslant-v / 2 .
$$

To find the critical curves $v_{c}(\phi)$ separating the regions of triad interactions with $S_{E}<1$ and $S_{E}>1$, we set $S_{E}=1$, obtaining

$$
\left(v_{c}^{2}+2 v_{c} \cos \phi+1\right)^{\alpha / 2}=2-v_{c}^{\alpha} .
$$

Note that $S_{E}<1$ for $v<v_{c}$ and $S_{E}>1$ for $v>v_{c}$. For $\alpha=1$, we obtain

$$
v_{c}(\phi)=\frac{3}{2 \cos \phi+4},
$$

while for $\alpha=2$, we obtain

$$
v_{c}(\phi)=\frac{-\cos \phi+\sqrt{\cos ^{2} \phi+2}}{2} .
$$

The critical curve is found implicitly for $\alpha=3$. 
For $S_{Z} \equiv X_{Z} / Y_{Z}$, we get

$$
S_{Z}=\left[\frac{v^{2}}{v^{2}+2 v \cos \phi+1}\right]^{\alpha / 2} \frac{\left(v^{2}+2 v \cos \phi+1\right)^{\alpha / 2}-1}{1-v^{\alpha}},
$$

with the critical curve $v_{c}(\phi)$ given implicitly by

$$
1=\left[\frac{v_{c}^{2}}{v_{c}^{2}+2 v_{c} \cos \phi+1}\right]^{\alpha / 2} \frac{\left(v_{c}^{2}+2 v_{c} \cos \phi+1\right)^{\alpha / 2}-1}{1-v_{c}^{\alpha}} .
$$

\section{Appendix C}

We follow the standard EDQNM procedure, see e.g. Leith (1971) and Leslie (1973), but start from the vorticity equation (1.1) instead of the velocity equation. Multiplying the viscous version of (4.3) for $\hat{\theta}(\boldsymbol{k}), \hat{\theta}(-\boldsymbol{p})$, and $\hat{\theta}(-\boldsymbol{q})$ by $\hat{\theta}(-\boldsymbol{p}, t) \hat{\theta}(-\boldsymbol{q}, t), \hat{\theta}(\boldsymbol{k}, t) \hat{\theta}(-\boldsymbol{q}, t)$, and $\hat{\theta}(\boldsymbol{k}, t) \hat{\theta}(-\boldsymbol{p}, t)$ respectively, we obtain an equation for $\hat{\theta}(\boldsymbol{k}, t) \hat{\theta}(-\boldsymbol{p}, t) \hat{\theta}(-\boldsymbol{q}, t)$,

$$
\begin{aligned}
{\left[\frac{\partial}{\partial t}+v\left(k^{2}+p^{2}+q^{2}\right)\right] \hat{\theta}(\boldsymbol{k}, t) \hat{\theta}(-\boldsymbol{p}, t) \hat{\theta}(-\boldsymbol{q}, t)=} & \frac{1}{2} \sum_{\boldsymbol{k}=\boldsymbol{r}+\boldsymbol{s}} \frac{\left(s^{\alpha}-r^{\alpha}\right)(\boldsymbol{r} \times \boldsymbol{s})_{z}}{r^{\alpha} S^{\alpha}} \delta_{\boldsymbol{r}+\boldsymbol{s}-\boldsymbol{k}} \\
& \times[\hat{\theta}(-\boldsymbol{p}, t) \hat{\theta}(-\boldsymbol{q}, t) \hat{\theta}(\boldsymbol{r}, t) \hat{\theta}(\boldsymbol{s}, t)] \\
& +\{\boldsymbol{k} \leftrightarrow-\boldsymbol{p}\}+\{\boldsymbol{k} \leftrightarrow-\boldsymbol{q}\}, \quad(\mathrm{C}
\end{aligned}
$$

and similarly an equation for $\hat{\theta}(-\boldsymbol{k}, t) \hat{\theta}(\boldsymbol{p}, t) \hat{\theta}(\boldsymbol{q}, t)$. The fourth moment is

$$
Q(\boldsymbol{k}, \boldsymbol{p}, \boldsymbol{q}, \boldsymbol{r})=\lim _{V \rightarrow 0} V^{-3}\langle\hat{\theta}(\boldsymbol{k}) \hat{\theta}(\boldsymbol{p}) \hat{\theta}(\boldsymbol{q}) \hat{\theta}(\boldsymbol{r})\rangle .
$$

Homogeneity requires $\boldsymbol{k}+\boldsymbol{p}+\boldsymbol{q}=0$ in the third moment and $\boldsymbol{k}+\boldsymbol{p}+\boldsymbol{q}+\boldsymbol{r}=0$ in the fourth moment. See McComb (1991), p. 56-57.

The evolution equations for the second and third moments are

$$
\begin{aligned}
\left(\frac{\partial}{\partial t}+2 v k^{2}\right) \Theta(\boldsymbol{k})= & \frac{1}{2} \int \mathrm{d} \boldsymbol{p} \mathrm{d} \boldsymbol{q} \delta^{2}(\boldsymbol{p}+\boldsymbol{q}-\boldsymbol{k}) \frac{\left(q^{\alpha}-p^{\alpha}\right)(\boldsymbol{p} \times \boldsymbol{q})_{z}}{p^{\alpha} q^{\alpha}} \\
& \times[\mathscr{R}(-\boldsymbol{k}, \boldsymbol{p}, \boldsymbol{q})+\mathscr{R}(\boldsymbol{k},-\boldsymbol{p},-\boldsymbol{q})] \\
= & k^{\alpha} T_{E}(\boldsymbol{k})
\end{aligned}
$$

and

$$
\begin{aligned}
{\left[\frac{\partial}{\partial t}+v\left(k^{2}+p^{2}+q^{2}\right)\right] \mathscr{R}(\boldsymbol{k},-\boldsymbol{p},-\boldsymbol{q})=} & \frac{1}{2} \int \mathrm{d} \boldsymbol{r} \frac{\left(s^{\alpha}-r^{\alpha}\right)(\boldsymbol{r} \times \boldsymbol{s})_{z}}{r^{\alpha} s^{\alpha}} \\
& \times\left[\delta^{2}(\boldsymbol{k}-\boldsymbol{r}-\boldsymbol{s}) Q(-\boldsymbol{p},-\boldsymbol{q}, \boldsymbol{r}, \boldsymbol{s})\right. \\
& +\{\boldsymbol{k} \leftrightarrow-\boldsymbol{p}\}+\{\boldsymbol{k} \leftrightarrow-\boldsymbol{q}\}] .
\end{aligned}
$$

At this point we close the hierarchy by discarding the fourth-order cumulant, i.e. we assume the fourth moment is related to the second moment as it would be for a Gaussian distribution. For the first term of (C4), we have

$$
\begin{aligned}
Q(-\boldsymbol{p},-\boldsymbol{q}, \boldsymbol{r}, \boldsymbol{s})= & \langle\hat{\theta}(-\boldsymbol{p}) \hat{\theta}(-\boldsymbol{q}) \hat{\theta}(\boldsymbol{r}) \hat{\theta}(\boldsymbol{s})\rangle \\
= & \langle\hat{\theta}(-\boldsymbol{p}) \hat{\theta}(-\boldsymbol{q})\rangle\langle\hat{\theta}(\boldsymbol{r}) \hat{\theta}(\boldsymbol{s})\rangle+\langle\hat{\theta}(-\boldsymbol{p}) \hat{\theta}(\boldsymbol{r})\rangle\langle\hat{\theta}(-\boldsymbol{q}) \hat{\theta}(\boldsymbol{s})\rangle \\
& +\langle\hat{\theta}(-\boldsymbol{p}) \hat{\theta}(\boldsymbol{s})\rangle\langle\hat{\theta}(-\boldsymbol{q}) \hat{\theta}(\boldsymbol{r})\rangle .
\end{aligned}
$$


Consider the first term of (C5), $\langle\hat{\theta}(-\boldsymbol{p}) \hat{\theta}(-\boldsymbol{q})\rangle\langle\hat{\theta}(\boldsymbol{r}) \hat{\theta}(\boldsymbol{s})\rangle$. By virtue of homogeneity, we have $\boldsymbol{p}+\boldsymbol{q}=0$ and $\boldsymbol{p}+\boldsymbol{q}=\boldsymbol{r}+\boldsymbol{s}$, with $\boldsymbol{k}=\boldsymbol{p}+\boldsymbol{q}$ (See McComb (1991), p. 79.). This implies $\boldsymbol{k}=\boldsymbol{r}+\boldsymbol{s}=0, \boldsymbol{r} \|-\boldsymbol{s}$ and $\boldsymbol{r} \times \boldsymbol{k}=\boldsymbol{r} \times \boldsymbol{s}=0$. Hence, the geometrical prefactor is zero and this term does not contribute. Enforcing homogeneity in the other two terms gives, for the first term in the integrand of (C4),

$$
\frac{\left(q^{\alpha}-p^{\alpha}\right)(\boldsymbol{p} \times \boldsymbol{q})_{z}}{p^{\alpha} q^{\alpha}} \Theta(\boldsymbol{p}) \Theta(\boldsymbol{q}) \delta^{2}(\boldsymbol{k}-\boldsymbol{p}-\boldsymbol{q}) .
$$

We carry out similar calculations for the other two terms, and repeat the procedure for the fourth moments in the equation for $\mathscr{R}(-\boldsymbol{k}, \boldsymbol{p}, \boldsymbol{q})$. Integrating then results in

$$
\begin{aligned}
& \mathscr{R}(-\boldsymbol{k}, \boldsymbol{p}, \boldsymbol{q})+\mathscr{R}(\boldsymbol{k},-\boldsymbol{p},-\boldsymbol{q})=2 \int_{0}^{t} \mathrm{~d} s \mathrm{e}^{\left\{-\left[v\left(k^{2}+p^{2}+q^{2}\right)+\mu_{k p q}\right](t-s)\right\}} \delta^{2}(\boldsymbol{k}-\boldsymbol{p}-\boldsymbol{q}) \\
& \quad \times\left[\frac{\left(q^{\alpha}-p^{\alpha}\right)(\boldsymbol{p} \times \boldsymbol{q})_{z}}{p^{\alpha} q^{\alpha}} \Theta(\boldsymbol{p}) \Theta(\boldsymbol{q})+\frac{\left(k^{\alpha}-q^{\alpha}\right)(\boldsymbol{p} \times \boldsymbol{q})_{z}}{k^{\alpha} q^{\alpha}} \Theta(\boldsymbol{k}) \Theta(\boldsymbol{q})\right. \\
& \left.-\frac{\left(k^{\alpha}-p^{\alpha}\right)(\boldsymbol{p} \times \boldsymbol{q})_{z}}{k^{\alpha} p^{\alpha}} \Theta(\boldsymbol{k}) \Theta(\boldsymbol{p})\right],
\end{aligned}
$$

where we have added an eddy damping rate $\mu_{k p q}$ to the viscous dissipation to represent the effect of the fourth-order cumulants on the third moment. We insert (C7) into (C 3), neglect viscous dissipation on the left-hand side since we are interested in inertial ranges, assume isotropy, and use the relation

$$
\mathscr{E}(k)=\pi k^{1-\alpha} \Theta(k) .
$$

We also carry out the time integral, assuming the characteristic time $\left[\mu_{k p q}+v\left(k^{2}+p^{2}+q^{2}\right)\right]^{-1}$ of the exponential is much shorter than the large-eddy turnover time characteristic of the terms inside the large square brackets (Lesieur 1993), and neglecting the time variation of $\mu_{k p q}$. This Markovianization, together with use of an eddy damping, ensures realizability, i.e. positivity of the energy spectrum (Orszag 1970, 1977). This yields

$$
\begin{aligned}
\left(\frac{\partial}{\partial t}\right. & \left.+2 v k^{2}\right) \mathscr{E}(k)=\frac{1}{\pi} \int \mathrm{d} \boldsymbol{p} \mathrm{d} \boldsymbol{q} \delta^{2}(\boldsymbol{p}+\boldsymbol{q}-\boldsymbol{k}) \\
& \times \frac{\left\{1-\mathrm{e}^{-\left[\mu_{k p q}+v\left(k^{2}+p^{2}+q^{2}\right) t\right]}\right\}}{\mu_{k p q}+v\left(k^{2}+p^{2}+q^{2}\right)} \frac{\left(q^{\alpha}-p^{\alpha}\right)\left(1-x^{2}\right)}{(k p q)^{\alpha-1}} \\
& \times\left[\left(q^{\alpha}-p^{\alpha}\right) \mathscr{E}(p) \mathscr{E}(q)+\left(k^{\alpha}-q^{\alpha}\right) \frac{p}{k} \mathscr{E}(k) \mathscr{E}(q)-\left(k^{\alpha}-p^{\alpha}\right) \frac{q}{k} \mathscr{E}(k) \mathscr{E}(p)\right],
\end{aligned}
$$

where

$$
x=\frac{\boldsymbol{p} \cdot \boldsymbol{q}}{p q}=\frac{p^{2}+q^{2}-k^{2}}{2 p q} .
$$

The integral on the right-hand side of (C9) is of the form

$$
I(k)=\int \mathrm{d} \boldsymbol{p} \mathrm{d} \boldsymbol{q} f(k, p, q) \delta^{2}(\boldsymbol{k}-\boldsymbol{p}-\boldsymbol{q}) .
$$

This 'bipolar integral' can be done using appropriate variable transformations. The procedure for three-dimensional turbulence is given in the appendix of Leslie (1973). 
For two dimensions, first align $\boldsymbol{k}$ with the $x$-axis and transform to polar coordinates:

$$
\begin{aligned}
& p_{x}=p \cos \beta, \quad p_{y}=p \sin \beta, \\
& q_{x}=q \cos \phi, \quad q_{y}=q \sin \phi,
\end{aligned}
$$

where $\beta$ is the angle between $\boldsymbol{p}$ and the positive $x$-axis (parallel to $\boldsymbol{k}$ ) and $\phi$ is the angle between $\boldsymbol{q}$ and $\boldsymbol{k}$ in the triad $\boldsymbol{k}, \boldsymbol{p}, \boldsymbol{q}$. The integral is then

$$
\begin{aligned}
I(k)= & \int_{0}^{\infty} \mathrm{d} p \int_{|k-p|}^{k+p} \mathrm{~d} q p q f(k, p, q) \\
& \times \int_{0}^{2 \pi} \int_{0}^{2 \pi} \mathrm{d} \beta \mathrm{d} \phi \delta(k-p \cos \beta-q \cos \phi) \delta(p \sin \beta+q \sin \phi) .
\end{aligned}
$$

Change variables to

$$
\begin{aligned}
& \xi=p \cos \beta+q \cos \phi, \\
& \eta=p \sin \beta+q \sin \phi .
\end{aligned}
$$

The Jacobian of the transformation is

$$
\frac{\partial(\xi, \eta)}{\partial(\beta, \phi)}=p q \sin (\phi-\beta)=p q \sin (-\gamma),
$$

where $\gamma$ is the angle between $\boldsymbol{p}$ and $\boldsymbol{q}$ in the triad $\boldsymbol{k}, \boldsymbol{p}, \boldsymbol{q}$. The integral then becomes

$$
\begin{aligned}
I(k) & =4 \int_{0}^{\infty} \mathrm{d} p \int_{|k-p|}^{k+p} \mathrm{~d} q f(k, p, q) \int_{-(p+q)}^{p+q} \int_{-(p+q)}^{p+q} \mathrm{~d} \xi \mathrm{d} \eta \frac{\delta(k-\xi) \delta(\eta)}{|\sin \gamma|} \\
& =2 \int_{0}^{\infty} \mathrm{d} p \int_{|k-p|}^{k+p} \mathrm{~d} q \frac{f(k, p, q)}{\sqrt{1-x^{2}}}
\end{aligned}
$$

where $x=\cos \gamma$,

$$
\int_{-(p+q)}^{p+q} \mathrm{~d} \eta \delta(\eta)=2 H(p+q)-1,
$$

and the properties of the Heaviside function

$$
H(x)= \begin{cases}0, & x<0 \\ 1 / 2, & x=0 \\ 1, & x>0\end{cases}
$$

have been used. Note that the expression given in Kraichnan (1967) for the bipolar integral is wrong by a factor of $\pi$.

Returning to (C 9) and using (C 18), after some algebra, we obtain

$$
\begin{aligned}
T_{E}(k)= & \frac{1}{\pi} \int_{0}^{\infty} \mathrm{d} p \int_{|k-p|}^{k+p} \mathrm{~d} q D_{k p q} \\
& \times \frac{k^{2}}{p q}\left[2 a_{k p q} k \mathscr{E}(p) \mathscr{E}(q)-b_{k p q} p \mathscr{E}(k) \mathscr{E}(q)-b_{k q p} q \mathscr{E}(k) \mathscr{E}(p)\right] \\
= & \frac{1}{2} \int_{0}^{\infty} \mathrm{d} p \int_{|k-p|}^{k+p} \mathrm{~d} q T_{E}(k, p, q),
\end{aligned}
$$


where $a_{k p q}$ and $b_{k p q}$ are as defined in (5.6), and we have taken $t \rightarrow \infty$ and $v=0$ in (C 9), whereupon the eddy damping becomes $\mu_{k p q}^{-1}=D_{k p q}$ defined in (5.8).

\section{Appendix D}

We write the flux past $k$ as

$$
\pi_{E}(k)=\int_{k}^{\infty} T_{E}\left(k^{\prime}\right) \mathrm{d} k^{\prime}=\frac{1}{2} \int_{k}^{\infty} \mathrm{d} k^{\prime} \int_{0}^{\infty} \int_{0}^{\infty} T_{E}\left(k^{\prime}, p, q\right) \mathrm{d} p \mathrm{~d} q,
$$

and break up the $p$ integral at $p=k$ :

$$
\begin{aligned}
\pi_{E}(k)= & \frac{1}{2} \int_{k}^{\infty} \mathrm{d} k^{\prime} \int_{0}^{k} \mathrm{~d} p \int_{0}^{\infty} \mathrm{d} q T_{E}\left(k^{\prime}, p, q\right) \\
& +\frac{1}{2} \int_{k}^{\infty} \mathrm{d} k^{\prime} \int_{k}^{\infty} \mathrm{d} p \int_{0}^{\infty} \mathrm{d} q T_{E}\left(k^{\prime}, p, q\right) .
\end{aligned}
$$

We then break up the $q$ integral at $q=k$, yielding four terms:

$$
\begin{aligned}
\pi_{E}(k)= & \frac{1}{2} \int_{k}^{\infty} \mathrm{d} k^{\prime} \int_{0}^{k} \mathrm{~d} p \int_{0}^{k} \mathrm{~d} q T_{E}\left(k^{\prime}, p, q\right) \\
& +\frac{1}{2} \int_{k}^{\infty} \mathrm{d} k^{\prime} \int_{0}^{k} \mathrm{~d} p \int_{k}^{\infty} \mathrm{d} q T_{E}\left(k^{\prime}, p, q\right) \\
& +\frac{1}{2} \int_{k}^{\infty} \mathrm{d} k^{\prime} \int_{k}^{\infty} \mathrm{d} p \int_{0}^{k} \mathrm{~d} q T_{E}\left(k^{\prime}, p, q\right) \\
& +\frac{1}{2} \int_{k}^{\infty} \mathrm{d} k^{\prime} \int_{k}^{\infty} \mathrm{d} p \int_{k}^{\infty} \mathrm{d} q T_{E}\left(k^{\prime}, p, q\right) .
\end{aligned}
$$

Relabelling dummy variables, we bring the second term into the form

$$
\begin{aligned}
\frac{1}{2} \int_{k}^{\infty} \mathrm{d} k^{\prime} \int_{0}^{k} \mathrm{~d} p \int_{k}^{\infty} \mathrm{d} q T_{E}\left(k^{\prime}, p, q\right) & =\frac{1}{2} \int_{k}^{\infty} \mathrm{d} k^{\prime} \int_{0}^{k} \mathrm{~d} q \int_{k}^{\infty} \mathrm{d} p T_{E}\left(k^{\prime}, q, p\right) \\
& =\frac{1}{2} \int_{k}^{\infty} \mathrm{d} p \int_{0}^{k} \mathrm{~d} q \int_{k}^{\infty} \mathrm{d} k^{\prime} T_{E}\left(p, q, k^{\prime}\right) .
\end{aligned}
$$

We add this to the third term of (D 3$)$ and use (4.5a) to obtain

$$
\begin{aligned}
& \frac{1}{2} \int_{k}^{\infty} \mathrm{d} k^{\prime} \int_{0}^{k} \mathrm{~d} q \int_{k}^{\infty} \mathrm{d} p T_{E}\left(p, q, k^{\prime}\right)+\frac{1}{2} \int_{k}^{\infty} \mathrm{d} k^{\prime} \int_{k}^{\infty} \mathrm{d} p \int_{0}^{k} \mathrm{~d} q T_{E}\left(k^{\prime}, p, q\right) \\
& \quad=\frac{1}{2} \int_{k}^{\infty} \mathrm{d} k^{\prime} \int_{k}^{\infty} \mathrm{d} p \int_{0}^{k} \mathrm{~d} q\left[T_{E}\left(p, q, k^{\prime}\right)+T_{E}\left(k^{\prime}, p, q\right)\right] \\
& \quad=\frac{1}{2} \int_{k}^{\infty} \mathrm{d} k^{\prime} \int_{k}^{\infty} \mathrm{d} p \int_{0}^{k} \mathrm{~d} q\left[-T_{E}\left(q, k^{\prime}, p\right)\right] \\
& =\frac{1}{2} \int_{0}^{k} \mathrm{~d} k^{\prime} \int_{k}^{\infty} \mathrm{d} p \int_{k}^{\infty} \mathrm{d} q\left[-T_{E}\left(k^{\prime}, p, q\right)\right],
\end{aligned}
$$

where we have relabelled dummy variables in the fourth line and note that the first argument of $T_{E}$ is integrated from 0 to $k$ in both the third and fourth lines of (D 5). We now show, relabelling dummy variables, switching the order of integration, and using 
(4.5a), that the fourth term of (D 3) vanishes:

$$
\begin{aligned}
\frac{1}{2} & \int_{k}^{\infty} \mathrm{d} k^{\prime} \int_{k}^{\infty} \mathrm{d} p \int_{k}^{\infty} \mathrm{d} q T_{E}\left(k^{\prime}, p, q\right) \\
= & \frac{1}{6} \int_{k}^{\infty} \mathrm{d} k^{\prime} \int_{k}^{\infty} \mathrm{d} p \int_{k}^{\infty} \mathrm{d} q T_{E}\left(k^{\prime}, p, q\right)+\frac{1}{6} \int_{k}^{\infty} \mathrm{d} p \int_{k}^{\infty} \mathrm{d} q \int_{k}^{\infty} \mathrm{d} k^{\prime} T_{E}\left(p, q, k^{\prime}\right) \\
& +\frac{1}{6} \int_{k}^{\infty} \mathrm{d} q^{\prime} \int_{k}^{\infty} \mathrm{d} k^{\prime} \int_{k}^{\infty} \mathrm{d} p T_{E}\left(q, k^{\prime}, p\right) \\
= & \frac{1}{6} \int_{k}^{\infty} \mathrm{d} k^{\prime} \int_{k}^{\infty} \mathrm{d} p \int_{k}^{\infty} \mathrm{d} q\left[T_{E}\left(k^{\prime}, p, q\right)+T_{E}\left(p, q, k^{\prime}\right)+T_{E}\left(q, k^{\prime}, p\right)\right] \\
= & 0 .
\end{aligned}
$$

Collecting the results, we obtain (5.14).

\section{Appendix E}

Let $p \leqslant k \leqslant q$. We wish to establish the sign of

$$
T_{E}(k, p, q)=\frac{2}{\pi} D_{k p q} \frac{k^{2}}{p q}\left[2 a_{k p q} k \mathscr{E}(p) \mathscr{E}(q)-b_{k p q} p \mathscr{E}(k) \mathscr{E}(q)-b_{k q p} q \mathscr{E}(k) \mathscr{E}(p)\right] .
$$

The following calculation is modelled on a similar one in the appendix of Kraichnan (1967). We assume a similarity scaling for $\mathscr{E}(k)$ :

$$
\mathscr{E}(p)=(p / k)^{-n} \mathscr{E}(k) .
$$

Let $p / k=v, q / k=w$, and substitute (E2) into (E 1) to obtain

$$
\begin{aligned}
T_{E}(k, p, q)= & \frac{4}{\pi} D_{k p q} \frac{k^{3}}{p q} a_{k p q}[\mathscr{E}(k)]^{2}(v w)^{-n} \\
& \times\left[1-\frac{\left(1-w^{\alpha}\right)}{\left(v^{\alpha}-w^{\alpha}\right)} v^{1+n}-\frac{\left(v^{\alpha}-1\right)}{\left(v^{\alpha}-w^{\alpha}\right)} w^{1+n}\right],
\end{aligned}
$$

where

$$
\begin{gathered}
b_{k p q} / a_{k p q}=2\left(q^{\alpha}-k^{\alpha}\right) /\left(q^{\alpha}-p^{\alpha}\right), \\
b_{k q p} / a_{k p q}=2\left(p^{\alpha}-k^{\alpha}\right) /\left(p^{\alpha}-q^{\alpha}\right)
\end{gathered}
$$

have been used. Note also that $a_{k p q} \geqslant 0$. The ordering assumption means $0 \leqslant v \leqslant 1$ and $1 \leqslant w \leqslant 1+v$.

Let

$$
K(v, w, n, \alpha)=1-v^{1+n} \frac{\left(1-w^{\alpha}\right)}{\left(v^{\alpha}-w^{\alpha}\right)}-w^{1+n} \frac{\left(v^{\alpha}-1\right)}{\left(v^{\alpha}-w^{\alpha}\right)} .
$$

The derivative with respect to $n$ is

$$
\begin{aligned}
\frac{\partial K(v, w, n, \alpha)}{\partial n} & =-v^{1+n} \ln (v) \frac{\left(w^{\alpha}-1\right)}{\left(w^{\alpha}-v^{\alpha}\right)}-w^{1+n} \ln (w) \frac{\left(1-v^{\alpha}\right)}{\left(w^{\alpha}-v^{\alpha}\right)} \\
& =\left[v^{1+n}\left(w^{\alpha}-1\right) \ln \left(v^{-1}\right)-w^{1+n}\left(1-v^{\alpha}\right) \ln (w)\right] /\left(w^{\alpha}-v^{\alpha}\right) .
\end{aligned}
$$

Fixing $v, w$ and $\alpha$, and using $0 \leqslant v \leqslant 1$ and $1 \leqslant w$, one sees that the first term is greater than or equal to zero and its magnitude decreases monotonically with $n$, while the second term is less than or equal to zero and of monotonically increasing 
magnitude. Hence, $\partial K(v, w, n, \alpha) / \partial n$ has one zero, and is positive as $n \rightarrow-\infty$ and negative as $n \rightarrow \infty$.

Here $K(v, w, n, \alpha)$ has zeros at $n=-1$ and $n=\alpha-1$. Therefore $K(v, w, n, \alpha)<0$ for $n<-1$ and $n>\alpha-1$, and $K(v, w, n, \alpha)>0$ for $-1<n<\alpha-1$. The KLB scaling is $n=(7-\alpha) / 3$, which for $n=\alpha-1$ yields

$$
\alpha-1=(7-\alpha) / 3 \Rightarrow \alpha=2.5,
$$

and for $n=-1$,

$$
-1=(7-\alpha) / 3 \Rightarrow \alpha=10 .
$$

At these values of $\alpha$ the KLB spectrum corresponds to equipartition and the transfers vanish, as shown in $\S 7$. For $2.5<\alpha<10, K(v, w,(7-\alpha) / 3, \alpha)>0$. Hence, transfer is into the middle wavenumber in the self-similar inertial range if the exponent for the energy spectrum falls between the limits set by enstrophy and energy equipartition, which it does for $2.5<\alpha<10$. On the other hand, transfer is out of the middle wavenumber in the self-similar inertial range for $0<\alpha<2.5$ and $\alpha>10$.

\section{REFERENCES}

BATCHELOR, G. K. 1959 Small-scale variation of convected quantities like temperature in turbulent fluid. Part 1. General discussion and the case of small conductivity. J. Fluid Mech. 5, 113-133.

BAtChelor, G. K. 1969 Computation of the energy spectrum in homogeneous two-dimensional turbulence. Phys. Fluids Suppl. II 12, 233-239.

Benzi, R., Vitaletti, M. \& Vulpiani, A. 1978 A variational principle for the statistical mechanics of fully developed turbulence. J. Phys. A: Math. Gen. 15, 883-895.

BLumen, W. 1978 Uniform potential vorticity flow. Part I. Theory of wave interactions and two-dimensional turbulence. J. Atmos. Sci. 35, 774-783.

Boffetta, G., De Lillo, F. \& Musacchio, S. 2002 Inverse cascade in Charney-HasegawaMima turbulence. Europhys. Lett. 59, 687-693.

Boffetta, G. \& Musacchio, S. 2010 Evidence for the double cascade scenario in twodimensional turbulence. Phys. Rev. E 82, 016307.

Borue, V. 1994 Inverse energy cascade in stationary two-dimensional homogeneous turbulence. Phys. Rev. Lett. 72, 1475-1478.

Bowman, J. C., Krommes, J. A. \& Ottaviani, M. 1993 The realizable Markovian closure. I. General theory, with application to three-wave dynamics. Phys. Fluids B 5, 3558-3589.

Carnevale, G. F., Frisch, U. \& Salmon, R. 1981 H theorems in statistical fluid dynamics. J. Phys. A: Math. Gen. 14, 1701-1718.

Chen, S., Ecke, R. E., Eyink, G. L., Rivera, M., Wan, M. \& Xiao, Z. 2006 Physical mechanism of the two-dimensional inverse energy cascade. Phys. Rev. Lett. 96, 084502.

Farazmand, M. M., Kevlahan, N. K.-R. \& Protas, B. 2011 Controlling the dual cascade of two-dimensional turbulence. J. Fluid Mech. 668, 202-222.

FJøRTOFT, R. 1953 On the changes in the spectral distribution of kinetic energy for two-dimensional non-divergent flow. Tellus 5, 225-230.

Fox, D. G. \& ORszag, S. A. 1973 Inviscid dynamics of two-dimensional turbulence. Phys. Fluids 16, 169-171.

Gkioulekas, E. \& Tung, K. K. 2007 A new proof on net upscale energy cascade in two-dimensional and quasi-geostrophic turbulence. J. Fluid Mech. 576, 173-189.

Held, I. M., Pierrehumbert, R. T., Garner, S. T. \& Swanson, K. L. 1995 Surface quasi-geostrophic dynamics. J. Fluid Mech. 282, 1-20.

Herring, J. R. \& MCWilliams, J. C. 1985 Comparison of direct numerical simulation of two-dimensional turbulence with two-point closure: the effects of intermittency. J. Fluid Mech. 153, 229-242. 
Iwayama, T., Shepherd, T. G. \& Watanabe, T. 2002 An 'ideal' form of decaying two-dimensional turbulence. J. Fluid Mech. 456, 183-198.

Iwayama, T. \& Watanabe, T. 2010 Green's function for a generalized two-dimensional fluid. Phys. Rev. E 82, 036307.

KRAICHNAN, R. H. 1958 a Irreversible statistical mechanics of incompressible hydromagnetic turbulence. Phys. Rev. 109, 1407-1422.

Kraichnan, R. H. $1958 b$ Higher order interactions in homogeneous turbulence theory. Phys. Fluids Rev. 1, 358-359.

KRAICHNAN, R. H. 1959 The structure of isotropic turbulence at very high Reynolds numbers. J. Fluid Mech. 5, 497-543.

Kraichnan, R. H. 1967 Inertial ranges in two-dimensional turbulence. Phys. Fluids 10, $1417-1423$.

Kraichnan, R. H. 1971a An almost-Markovian Galilean-invariant turbulence model. J. Fluid Mech. 47, 513-524.

Kraichnan, R. H. $1971 b$ Inertial-range transfer in two- and three-dimensional turbulence. J. Fluid Mech. 47, 525-535.

Kraichnan, R. H. 1975 Statistical dynamics of two-dimensional flow. J. Fluid Mech. 67, $155-175$.

Kraichnan, R. H. 1976 Eddy viscosity in two and three dimensions. J. Atmos. Sci. 33, $1521-1536$.

Kraichnan, R. H. \& Montgomery, D. 1980 Two-dimensionsional turbulence. Rep. Prog. Phys. 43, 547-619.

LARICHEV, V. D. \& MCWilliams, J. C. 1991 Weakly decaying turbulence in an equivalentbarotropic fluid. Phys. Fluids A 3, 938-950.

Leith, C. E. 1968 Diffusion approximation for two-dimensional turbulence. Phys. Fluids 11, 671-673.

Leith, C. E. 1971 Atmospheric predictability and two-dimensional turbulence. J. Atmos. Sci. 28, $145-161$.

LESIEUR, M. 1993 Turbulence in Fluids, 2nd edn. Kluwer.

LESLIE, D. C. 1973 Developments in the Theory of Turbulence. Oxford University Press.

Martin, P. C., Siggia, E. D. \& Rose, H. A. 1973 Statistical dynamics of classical systems. Phys. Rev. A 8, 423-437.

MсСомв, W. D. 1991 The Physics of Fluid Turbulence. Oxford University Press.

Merilees, P. E. \& WARN, H. 1975 On energy and enstrophy exchanges in two-dimensional non-divergent flow. J. Fluid Mech. 69, 625-630.

Orszag, S. A. 1970 Analytical theories of turbulence. J. Fluid Mech. 41, 363-386.

Orszag, S. A. 1977 Statistical Theory of Turbulence in Fluid Dynamics 1973 Les Houches Summer School of Theoretical Physics (ed. R. Balian \& J. L. Peube). Gordon and Breach.

PARET, J. \& TABEling, P. 1997 Experimental observation of the two-dimensional inverse energy cascade. Phys. Rev. Lett. 79, 4162.

PARET, J. \& TABELING, P. 1998 Intermittency in the two-dimensional inverse cascade of energy. Phys. Fluids 10, 3126-3136.

Pierrehumbert, R. T., Held, I. M. \& Swanson, K. L. 1994 Spectra of local and non-local two-dimensional turbulence. Chaos Solitons Fractals 4, 1111-1116.

RHINES, P. 1975 Waves and turbulence on a beta-plane. J. Fluid Mech. 69, 417-443.

Rhines, P. 1979 Geostrophic turbulence. Annu. Rev. Fluid Mech. 11, 401-441.

Salmon, R., Holloway, G. \& Hendershott, M. C. 1976 The equilibrium statistical mechanics of simple quasi-geostrophic models. J. Fluid Mech. 75, 691-703.

Salmon, R. 1998 Lectures on Geophysical Fluid Dynamics. Oxford University Press.

Schorghofer, N. 2000 Energy spectra of steady two-dimensional turbulent flows. Phys. Rev. E 61, 6572-6577.

SCOTT, R. K. 2007 Nonrobustness of the two-dimensional turbulent inverse cascade. Phys. Rev. E 75, 046301. 
Smith, K. S., Boccaletti, G., Henning, C. C., Marinov, I., Tam, C. Y., Held, I. M. \& VALLIS, G. K. 2002 Turbulent diffusion in the geostrophic inverse cascade. J. Fluid Mech. 469, 13-48.

Smith, L. M. \& Yакнот, V. 1994 Finite-size effects in forced two-dimensional turbulence. J. Fluid Mech. 274, 115-138.

Tran, C. V. 2004 Nonlinear transfer and spectral distribution of energy in $\alpha$ turbulence. Physica D 191, 137-155.

Tran, C. V., Dritschel, D. G. \& ScotT, R. K. 2010 Effective degrees of nonlinearity in a family of generalized models of two-dimensional turbulence. Phys. Rev. E 76, 046303.

Tung, K. \& Welch, W. 2001 Remarks on Charney's note on geostrophic turbulence. J. Atmos. Sci. 58, 2009-2012.

VALLGREN, A. 2011 Infrared Reynolds number dependency of two-dimensional inverse energy cascade. J. Fluid Mech. 667, 463-473.

VALLIS, G. K. 1985 Remarks on the predictability properties of two- and three-dimensional flow. Q. J. R. Meteorol. Soc. 111, 1039-1047.

VAllis, G. K. 2006 Atmospheric and Oceanic Fluid Dynamics. Cambridge University Press.

Watanabe, T. \& Iwayama, T. 2004 Unified scaling theory for local and non-local transfers in generalized two-dimensional turbulence. J. Phys. Soc. Japan 12, 3319-3330.

Watanabe, T. \& IWAyama, T. 2007 Interacting scales and triad enstrophy transfers in generalized two-dimensional turbulence. Phys. Rev. E 81, 016301.

Weinstein, S. A., Olson, P. L. \& Yuen, D. A. 1989 Time-dependent large aspect-ratio thermal convection in the Earth's mantle. Geophys. Astrophys. Fluid Dyn. 47, 157-197.

XiaO, Z., Wan, M., Chen, S. \& Eyink, G. L. 2009 Physical mechanism of the inverse energy cascade of two-dimensional turbulence: a numerical investigation. J. Fluid Mech. 619, 1-44. 